\title{
Modeling and Analysis of Emergency Messaging Delay in Vehicular Ad hoc Networks
}

\author{
by
}

Khadige Hussein Abboud

\author{
A thesis \\ presented to the University of Waterloo \\ in fulfillment of the \\ thesis requirement for the degree of \\ Master of Applied Science \\ in \\ Electrical and Computer Engineering
}

Waterloo, Ontario, Canada, 2009

CKhadige Hussein Abboud 2009 
I hereby declare that I am the sole author of this thesis. This is a true copy of the thesis, including any required final revisions, as accepted by my examiners.

I understand that my thesis may be made electronically available to the public. 


\begin{abstract}
Road crashes, occurring at a high annual rate for many years, demand improvements in transportation systems to provide a high level of on-road safety. Implanting smart sensors, communication capabilities, memory storage and information processing units in vehicles are important components of Intelligent Transportation Systems (ITS). ITS should enable the communication between vehicles and allow cooperative driving and early warnings of sudden breaks and accidents ahead. The prompt availability of the emergency information will provide the driver a time to react in order to avoid possible accidents ahead. Hence, information delivery delay is an importance quality-of-service (QoS) metric in such applications. In this thesis, we focus on modeling the delay for emergency messaging in vehicular ad hoc networks (VANETs). VANETs consist of nodes moving with very high speeds, resulting in frequent topological changes. As a result, many existing models and packet forwarding schemes designed for general purpose mobile ad hoc networks (MANETs) cannot be directly applied to VANETs.
\end{abstract}

In our system model, we consider mobility and traffic density of vehicles. We focus on studying the effect of the traffic flow density on the delay of emergency message dissemination. Hence, traffic flow theories developed by civil engineers form the base of our modeling.

The common way of emergency message dissemination in VANETs is broadcasting. To overcome the broadcasting storm problem and improve scalability of such large networks, we adopt a node cluster based broadcasting mechanism. This research provides a realistic 
mathematical model for the broadcasting delay, which accounts for the randomness in user mobility and matches the highly dynamic nature of VANETs. An investigation on the minimum cluster size that achieves acceptable message delivery latency is provided. It is shown that network control and performance parameters are dependent on the traffic density. Experimental measurement data are used to demonstrate the accuracy of the mathematical modeling. 


\section{Acknowledgements}

This thesis would not be accomplished without the support of many people. I would like to express my deep gratitude to my supervisor, Prof. Weihua Zhuang, who presented great help and guidance throughout my studies. Her wide scope, clear vision and time devotion are things I have always admired. I would like to thank my committee, Prof. Wang Zhou and Prof. Sherman Shen, for their valuable comments and feedback. I highly appreciate their time and effort in reading my thesis. Gratitude is due to the National Science and Engineering Research Council (NSERC) of Canada and Ontario Graduate Scholarship (OGS) for a research grant and a scholarship, respectively that supported me during my study.

I also would like to thank Prof. Liping Fu, for his valuable discussions which had a big role in the base of this research. I would like to extend my gratitude to all members of the Broadband Communication Research (BBCR) group for their discussions and suggestions with special thanks to Dr. Mohamad Awad and Yipin Sun for their help and encouragement.

I hope I can be able to show my gratitude for my parents, who were and still are the reason behind my success. Their support, prayers, love, encouragement and efforts are things I can never pay back. Gratitude is also due to my brothers and sisters for their tolerance and help during those two years. I am so grateful to my friends and professors back in Kuwait University, who paved the road for me to come here. Special thanks to my dearest friend Afrah Lutfi, who has been supportive and caring all these years.

I wish I can express my deepest gratitude for my best friend and my fiancé, Ali-Akbar Samadani. I will not forget his stand beside me, his endless love, encouragement, patience 
and sacrifices across the miles. I am grateful for his being, for his existence in my life and understanding to our distance for the sake of education. 


\section{Dedication}

To faith, dignity and freedom

To the soil of my home land, Lebanon

To my parents, Hussein Abboud and Jamila Al-Amin

To my best friend and the love of my life, Ali-Akbar Samadani 


\section{Table of Contents}

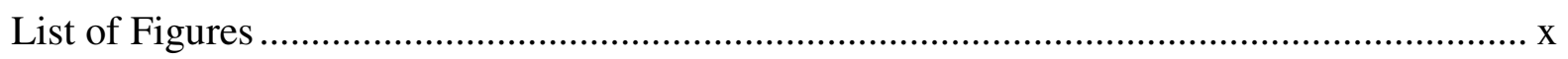

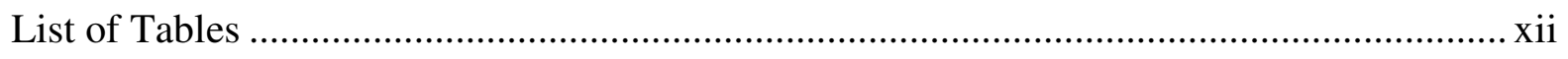

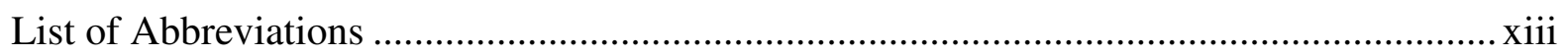

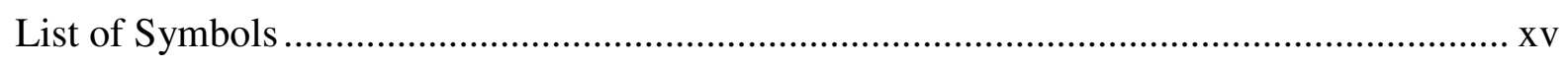

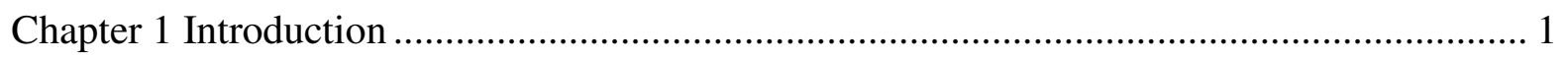

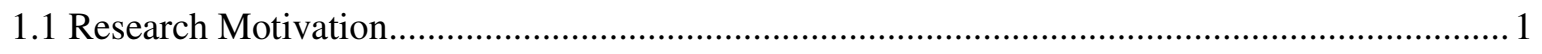

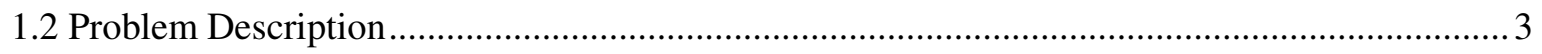

1.3 Research Objectives and Contributions.......................................................................

1.4 Thesis Structure …………………………………………………………………………5

Chapter 2 Overview of Vehicular Ad hoc Networks ........................................................... 6

2.1 Vehicular Ad hoc Networks and Applications ...................................................................... 6

2.1.1 Challenges in VANETs ...............................................................................................

2.2 Node Clustering in Wireless ad hoc Networks................................................................

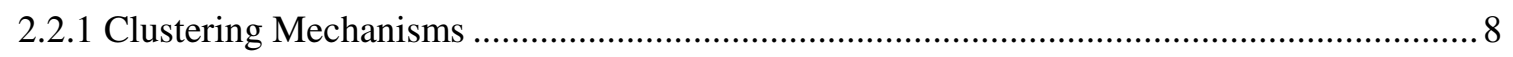

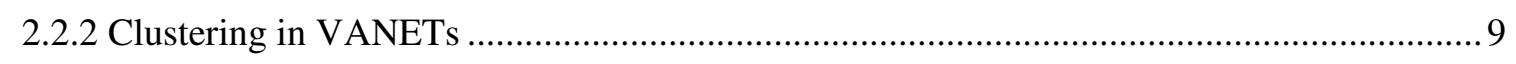

2.3 Message Dissemination in VANETs ............................................................................. 10

2.4 The Communication Channel......................................................................................... 13

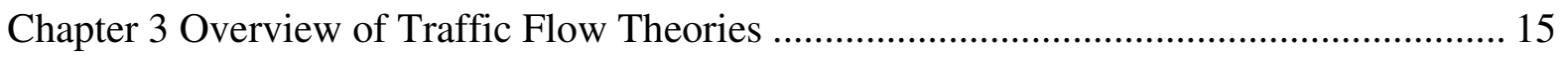

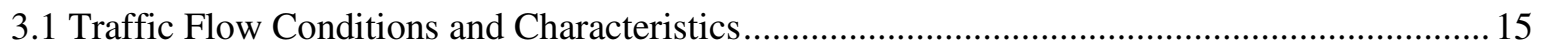

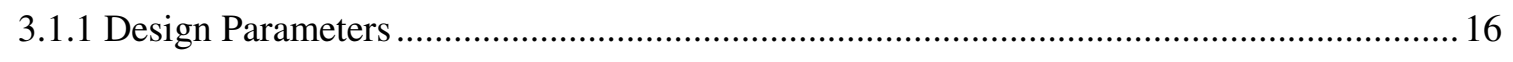

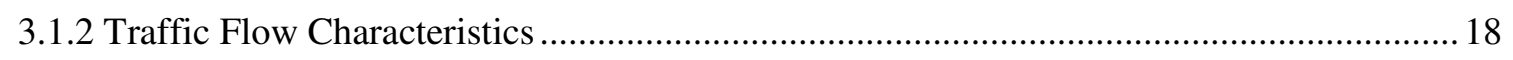

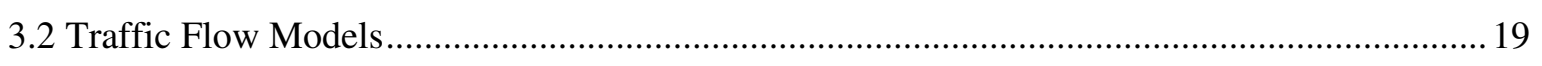

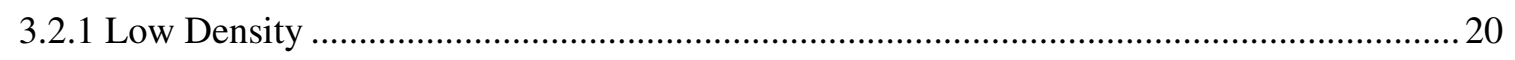

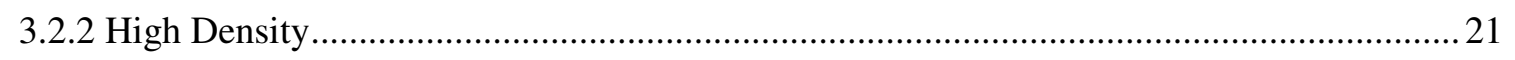

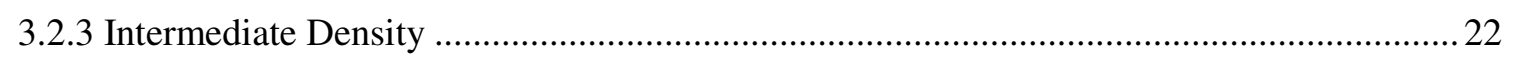

Chapter 4 Modeling and Analysis for Cluster based Emergency Broadcasting Delay .......... 25 


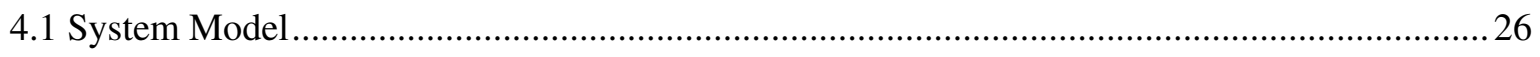

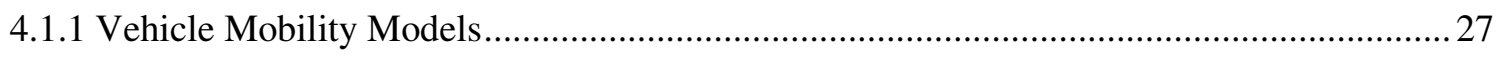

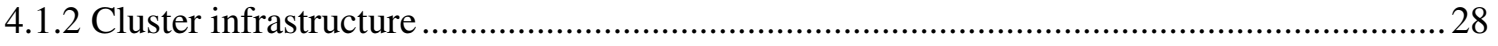

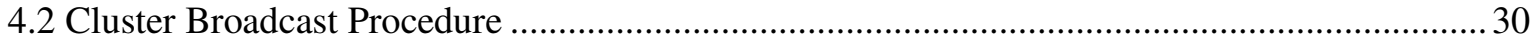

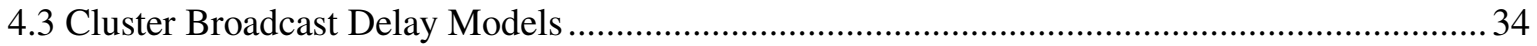

4.4 Numerical Analysis and Simulation Results ........................................................................ 36

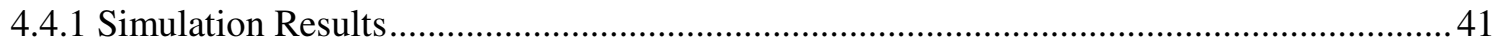

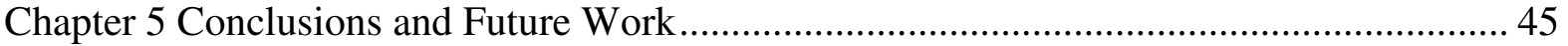

5.1 Conclusions

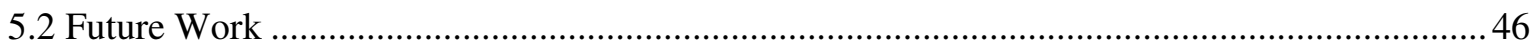

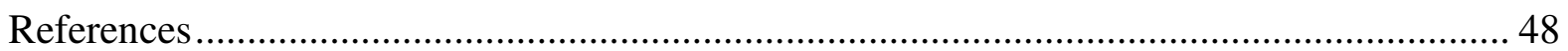




\section{List of Figures}

Figure 1. Pile-up on a freeway caused by non availability of the time required for vehicles to slow down

Figure 2. Types of node clustering, (a) non-overlapping, (b) common gateway used for inter cluster communication, (c) direct communication between adjacent $\mathrm{CHs}$ 9

Figure 3. Collision warning broadcast in bidirectional highway using IVG protocol [25] .... 12 Figure 4. Emergency message dissemination in BROADCOMM [27], with the highway being partitioned into equal sized clusters ................................................................. 13

Figure 5. Layout of the U.S. 5.9GHz ITS frequency band [6] ........................................ 14

Figure 6. An illustration of the VANET under consideration .......................................... 27

Figure 7. Cluster formation process and gateways $\left(\mathrm{GW}_{\mathrm{i}}\right.$ and $\left.\mathrm{GW}_{\mathrm{o}}\right)$ selection .................... 29

Figure 8. Emergency broadcasting from a source node A through the backbone of the clustered infrastructure till the last node in ZOR ..................................................... 31

Figure 9. Mobility aware cluster-based broadcasting procedure ....................................... 32

Figure 10. The total broadcasting delay versus cluster size (represented by transmission range) for different traffic flow densities $(10,20,50$, and $80 \mathrm{veh} / \mathrm{ml} / \mathrm{lane})$....................... 38 Figure 11. The lower bound cluster size versus traffic flow density for the three traffic flow states

Figure 12. Total broadcasting delay versus traffic flow density for different transmission ranges $(330,660,2300$, and $3300 \mathrm{ft})$ 
Figure 13 Comparison between simulation results and numerical values for the total broadcasting delay versus cluster size (represented by transmission range) for a density of 30

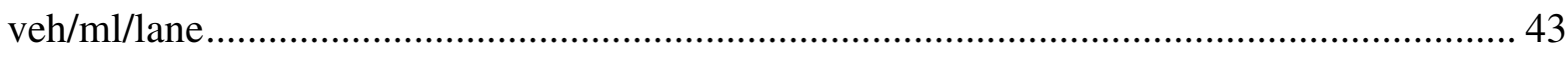




\section{List of Tables}

Table 1. Levels of service for basic freeway sections under ideal conditions and 70mpr

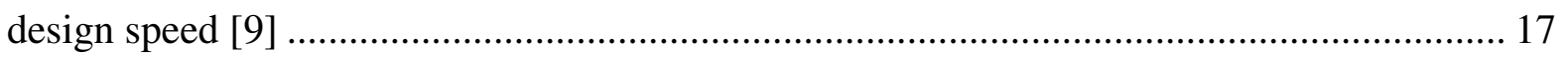

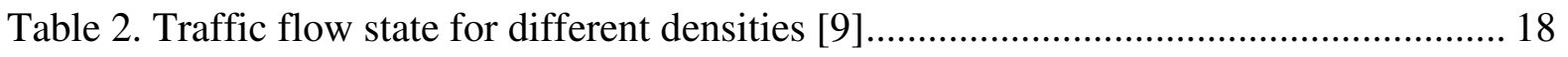

Table 3. Traffic flow models for different flow densities..................................................... 24

Table 4. List of the probability values required for modeling .............................................. 36 


\section{List of Abbreviations}

\begin{tabular}{|c|c|}
\hline AP & Access Point \\
\hline A-STAR & Anchor-based Street and Traffic Aware Routing \\
\hline $\mathrm{CCH}$ & Control Channel \\
\hline $\mathrm{CCW}$ & Cooperative Collision Warning \\
\hline $\mathrm{CDF}$ & Cumulative Distribution Function \\
\hline CFR & Cluster Formation Request \\
\hline $\mathrm{CH}$ & Cluster Head \\
\hline $\mathrm{CM}$ & Cluster Member \\
\hline DCA & Distributed Clustering Algorithm \\
\hline DFR & Design Flow Rate \\
\hline DSRC & Dedicated Short Range Communication \\
\hline GM & General Motors \\
\hline GPS & Global Positioning System \\
\hline GPSR & Greedy Perimeter Stateless Routing \\
\hline GSR & Geographic Source Routing \\
\hline ITS & Intelligent Transportation System \\
\hline IVC & Inter-vehicle Communication \\
\hline IVG & Inter-vehicle Geocast \\
\hline MAC & Medium Access Control \\
\hline MANET & Mobile Ad hoc Network \\
\hline
\end{tabular}




$\begin{array}{ll}\text { NGSIM } & \text { Next Generation Simulation } \\ \text { PDF } & \text { Probability Density Function } \\ \text { QoS } & \text { Quality of Service } \\ \text { SCH } & \text { Service Channel } \\ \text { VANET } & \text { Vehicular Ad hoc Network } \\ \text { V2I } & \text { Vehicle to Infrastructure } \\ \text { WAVE } & \text { Wireless Access in Vehicular Environment } \\ \text { WSN } & \text { Wireless Sensor Network } \\ \text { ZOR } & \text { Zone of Relevance }\end{array}$




\section{List of Symbols}

\author{
$\alpha$ \\ $\gamma$ \\ $\lambda_{t}$ \\ $\lambda_{s}$ \\ $\mu_{p}$ \\ $\bar{v}$ \\ $\sigma$ \\ $\tau$ \\ $\Phi$ \\ $c$ \\ $c_{j}$ \\ $C_{g}$ \\ $C_{o, I}$ \\ $D$ \\ $D_{o, I}$ \\ $D_{p}$ \\ $f_{H V}$ \\ $f_{p}$ \\ Minimum allowed distance headway \\ Constant for the truncated normal time headway distribution \\ Per lane Vehicle arrival rate \\ Per lane vehicle arrival rate in terms of distance \\ Average time headway for vehicles travelling in platoons \\ Space mean speed \\ Constant for the truncated normal distance headway distribution \\ Standard deviation \\ Minimum allowable time headway \\ Cumulative distribution function of the standard normal distribution \\ Road Capacity \\ Road capacity under ideal traffic conditions \\ Event that two consecutive clusters are segregated \\ Event that the two neighboring gateways of consecutive clusters are connected \\ Traffic flow density \\ Event that the two neighboring gateways of consecutive clusters are \\ disconnected \\ Density threshold above which all vehicles are assumed to be in platoons \\ Per lane heavy vehicle factor \\ Per lane driver population factors
}




$\begin{array}{ll}f_{w} & \text { Per lane width factor } \\ G_{A} & \text { Event that a gateway is available } \\ G_{N A} & \text { Event that a gateway is not available } \\ G W_{i} & \text { Gateway responsible for intra-cluster broadcast } \\ G W_{o} & \text { Gateway responsible for inter-cluster broadcast } \\ k & \text { Number of nodes between cluster head and gateway } \\ L & \text { The distance between the cluster head and gateway } \\ M_{i} & \text { Event that } G W_{i} \text { has moved closer to its CH } \\ M_{o} & \text { Event that } G W_{o} \text { has moved closer to its CH } \\ N & \text { Number of lanes on a highway } \\ N_{c} & \text { Number of clusters on a highway } \\ P_{N P} & \text { Fraction of vehicles driving not in platoons } \\ P_{p} & \text { Fraction of vehicles traveling in platoons } \\ R & \text { Transmission Range } \\ T_{D I} & \text { Total broadcasting delay for intermediate traffic flow density } \\ S_{N P} & \text { Standard deviation for vehicles not in platoons } \\ S_{p} & \text { Standard deviation for vehicles traveling in platoons } \\ S & \text { Distance headway } \\ T_{D} & \text { Timeadway }\end{array}$




$\begin{array}{ll}T_{D L} & \text { Total broadcasting delay for low traffic flow density } \\ T_{D R} & \text { Total broadcasting delay considering traffic movement } \\ T_{D S} & \text { Total broadcasting delay for a static infrastructure } \\ T_{E} & \text { Total Gateway re-election time } \\ T_{G W i} & \text { Time delay of the intra-cluster broadcast of the gateway } \\ T_{G W o} & \text { Time delay of the inter-cluster broadcast of the gateway } \\ T_{H} & \text { Cluster segregation healing time } \\ T_{P} & \text { Maximal period of the cluster maintenance time } \\ T_{1 s t} & \text { Broadcast transmission delay of safety message from the source node } \\ \bar{T} & \text { Average time headway } \\ \bar{T}_{N P} & \text { Average time headway for vehicles not traveling in platoons. } \\ V & \text { Traffic Flow rate } \\ X & \text { Number of vehicles arriving to a highway in a time interval } x\end{array}$




\section{Chapter 1}

\section{Introduction}

\subsection{Research Motivation}

Road crashes causing losses in lives, health and property have been occurring for many years. Statistics show that motor vehicle crashes represent almost $36 \%$ and $9 \%$ of the annual rate of unintentional death and disabling injuries, respectively in America for year 2007 , which leads to economical losses of $\$ 257.7$ billion [1]. One of the major car accident types that contribute widely to the number of annual injuries is the multi-vehicle collision or so called pile-up. Pile-ups, as shown in Figure 1, are likely to occur on freeways, where vehicles are travelling with a high speed. If any vehicle encounters a problem, vehicles behind it may not have enough time to slow down for the appropriate relative speed causing, serial accidents. Pile-ups can vary to include couple of vehicles to more than a hundred. In July 2009, Germany recorded the largest pile-up in the area that involved 259 vehicles causing tens of injuries and estimated loss of $€ 1.5$ million [2].

Throughout the years, many efforts have been made to deploy communication capabilities in vehicles and the transport infrastructure, leading to intelligent transportation systems (ITS) [3]. It is envisioned that wireless communication will allow both inter-vehicle communication (IVC) and communication between vehicles and a fixed infrastructure (V2I), 


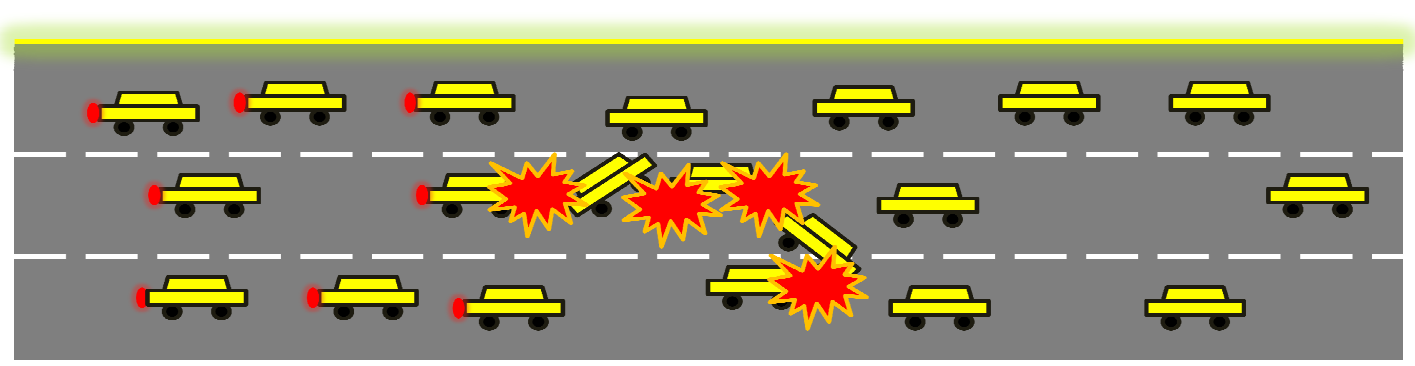

Figure 1. Pile-up on a freeway caused by non availability of the time required for vehicles to slow down

forming a vehicular ad hoc network (VANET) for exchanging messages carrying both safety and non-safety information. Currently, many safety applications are already available on road, such as Onstar's “Automatic Crash Response” project by General Motors (GM) [4]. Onstar makes use of V2I communications for an emergency advisor in a central room to receive messages from a crashed vehicle at the instant of incident, reporting details of the damage and therefore allowing immediate help in the accident spot. However such an application targets individual safety, while a catastrophic scenario occurs at pile-ups. Hence, it is important to allow warning of collision and sudden break among nearby vehicles. Accordingly, in this research, we adopt the emergent type (level 4 [5]) of cooperative collision warning $(\mathrm{CCW})$.

When on-board sensors detect an accident or a sudden break, the information should be carried via an emergency message to all vehicles in the area exposed to the potential danger, referred to as zone of relevance (ZOR), which extends behind the source vehicle along the highway. When such a sudden on-road phenomenon happens, the accumulated reaction time and vehicle processing delay give the shortest reaction time for the last vehicle in ZOR to avoid crashing with the leading vehicle. Thus, message delivery delay is a main 
quality-of-service (QoS) metrics for safety applications in VANETs. It is important to model the message dissemination delay along ZOR, which will help determining the system design parameters to satisfy the delay requirement for $\mathrm{CCW}$.

Existing mathematical models and analysis for mobile ad hoc networks (MANETs) are not valid for VANETs. Generally, message disseminating in VANETs is more challenging than that in MANETs, due to the high mobility level of vehicles on road, and the constraints imposed by road topology, movement direction, and speed limit. The high speeds and frequent network topology changes in VANETs affect the connectivity between nodes (vehicles) which can cause interruptions and require retransmissions. This research aims at providing a base model and analysis that take into account the effect of realistic traffic mobility on the message dissemination delay.

\subsection{Problem Description}

For $\mathrm{CCW}$, the best routing strategy to deliver a message to all the nodes in a certain area is broadcasting. The simplest way to broadcast a message is to allow every receiver to re-broadcast, which is known as flooding. However, with an increase in the number of nodes, packet collisions occur and delay increases due to retransmissions. In order to overcome the limitations of flooding and increase the scalability of the broadcasting, only particular nodes are chosen to relay the message. Many techniques have been proposed in literature for choosing the relay nodes, one of which makes use of the clustered network infrastructure where the backbone, consisting of cluster heads (CHs) and gateways, is used to relay messages $[6,27]$. 
The main focus of this work lies in studying the effect of traffic movement and mobility in modeling the broadcasting delay. Conventional mobility models such as random way point are not suitable for our study. For VANETs, some mobility models have been proposed in literature, mostly for a limited traffic condition. For example, a model for sparse networks is considered in [7], where each vehicle is assumed to move independently of the others, while [8] studies the two extremes of traffic flow density (sparse and dense). Both studies do not include the most commonly encountered scenario in reality, which lies between the two extremes. In this research, we use models that account for the mobility and randomness for all traffic flow density levels and that match the highly dynamic nature of a VANET. The models are based on the traffic flow theories developed in civil engineering [9], which suggests different mathematical models for different traffic densities.

\subsection{Research Objectives and Contributions}

The critical importance of delay as a QoS metric in CCW requires a mathematical model for it that builds the base for VANET performance analysis and network design. In this work, we aim to study the effect of traffic flow on the message propagation from vehicle to vehicle. We develop a mathematical model for the total delay in emergency message broadcasting based on the traffic flow theories given in [9] for three traffic flow levels (high, medium and low). In addition, we determine the minimum cluster size as a design requirement for the already existing clustered infrastructure upon which the broadcasting algorithm works, while the cluster size is upper bounded by other applications on highway. 


\subsection{Thesis Structure}

Safety applications such as CCW are some of many applications implemented or proposed for VANETs. The significant impact of the type of application on the protocol design and communication models in VANETs drives us to discuss the various applications and the imposed challenges in Chapter 2. An overview of VANETs is introduced in Chapter 2 to build the necessary background required for a reader to capture most of this research work. Since our study focuses on clustered VANETs, a literature survey on node clustering in wireless ad hoc networks is summarized. Chapter 3 includes an overview on the traffic flow theories developed in civil engineering as a base of our research. We introduce our work in Chapter 4, which includes 1) the system model and assumptions on the vehicle movement patterns and the clustered infrastructure, and 2) our cluster based broadcasting delay models with numerical analysis and simulation results. We conclude this research in Chapter 5, with a brief discussion on future work. 


\section{Chapter 2}

\section{Overview of Vehicular Ad hoc Networks}

This chapter includes a brief background, necessary for the understanding of this research. We start with a discussion of different applications and challenges in VANETs. This is followed by a literature survey about node clustering mechanisms in both MANETs and VANETs as a base for some message dissemination approaches used in safety applications. Finally we discuss the physical channels allocated for VANETs and some of its characteristics.

\subsection{Vehicular Ad hoc Networks and Applications}

Many dream applications can be enabled by intelligence added to transportation system using wireless communications technologies. ITS applications include two types, the comfort applications and safety applications [10]. Comfort ones include providing the driver with information about weather, maps and directions, locations for nearby petrol stations and restaurants, and available prices. Internet access and multimedia applications can be provided by V2I communication. On the other hand, safety applications are crucial for ensuring level of safety of the driver. This includes applications such as emergency warning, lane changing assistance, intersection coordination, which are mostly to be provided by IVC. 


\subsubsection{Challenges in VANETs}

The ad hoc nature of VANETs, which have no fixed infrastructure, makes VANET a subclass of MANET. However, VANETs do not inherit all characteristics of regular MANETs, and possess new challenges. Most challenges are due to the nature of traffic and the movement scenarios of vehicles [10-12]. For example, there are five main issues in networking properties of VANETs, which are rapid topology changes, frequent fragmentation, large network scale, variable network density and the geographical addressing that suits VANET applications $[10,11]$. Also there is a need for adaptive transmit power that achieves reliable and low latency transmission, and the penetration rate of the vehicular technology among vehicles imposes limitation on the market availability of many applications [13]. A careful study indicates that new challenges in VANETs are mainly due to the highly mobile nodes, constraints on the movement of vehicles, randomness of driver behavior, and the variable traffic density from time to time.

\subsection{Node Clustering in Wireless ad hoc Networks}

Different kinds of wireless ad hoc networks have immerged to support various applications, such as wireless sensor networks (WSNs), MANETs, and VANETs. The differences in nature of these networks introduce different constraints and challenges that need to be addressed in routing protocol design to achieve high performance. Yet, many applications involve a large number of nodes in these networks, thus, clustering a network into smaller manageable groups has served all these kinds of networks, for reducing energy 
consumption, improving resource utilization, balancing the load, reducing redundancy, retransmissions and collisions, or avoiding broadcasting storm.

\subsubsection{Clustering Mechanisms}

When designing a clustering scheme, the nature of the network and the application imposes emphasis on a certain objective from those previously mentioned. The choice of cluster formation metrics, such as the number of nodes per cluster, the size of the cluster, the number of clusters in the network, the transmission range, the cluster head election criteria, and the rule of forming a cluster, should all remain under the umbrella of the main predefined objective. Clustering a network is done by grouping nodes into groups each having a leader called cluster head $(\mathrm{CH})$ with other cluster members $(\mathrm{CMs})$ communicating only within the cluster (intra-cluster communication). Some clustering techniques require a border node or a gateway node to help connecting clusters together. A $\mathrm{CH}$ collects the data from its $\mathrm{CMs}$ and communicates with other $\mathrm{CHs}$ and gateways (inter-cluster communication) to deliver data to destination. Figure 2 shows different clustering techniques, in which two neighboring $\mathrm{CHs}$ communicate either directly, via shared gateways or using gateways from both clusters.

Partitioning the network into smaller manageable node clusters clearly supports scalability in all kinds of networks that have large number of nodes such as MANETs, WSNs, or VANETs. For WSNs, clustering can be used for increasing the life time of the network, either by balancing the load among nodes so as not to drain certain nodes or by assigning sleep schedules for $\mathrm{CMs}$ by $\mathrm{CHs}$. For example, the S-MAC protocol uses clustering for scheduling sleep periods [14], while the optimal cluster size that achieves the 


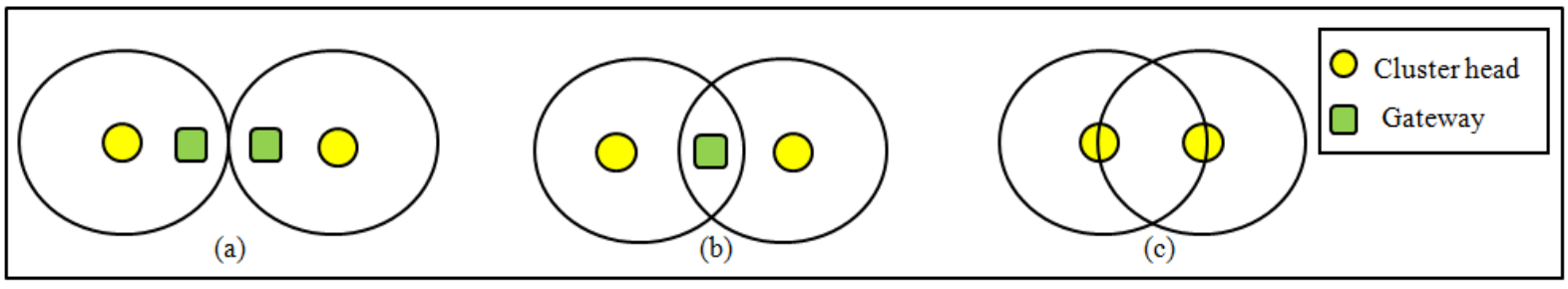

Figure 2. Types of node clustering, (a) non-overlapping, (b) common gateway used for inter cluster communication, (c) direct communication between adjacent $\mathrm{CHs}$

longest network lifetime can be determined according to the tradeoff between transmission range and number of CMs [15]. In MANETs, the mobility of nodes adds challenges for clustering in terms of frequent re-clustering and overhead of cluster maintenance message exchanges. The challenges are defined in [16] as clustering cost. This cost can be compared with the clustering profit and the system objective, which accordingly defines the significance of node clustering. For some MANETs, accounting for mobility in cluster formation is a key issue. MOBIC [17] suggests choosing a node with minimum relative speed from its neighbors as a $\mathrm{CH}$. This $\mathrm{CH}$ election criterion improves cluster stability and reduces the frequency of re-clustering due to node mobility.

\subsubsection{Clustering in VANETs}

As in MANETs and WSNs, clustering serves as a method to increase scalability and help network management of dense VANETs. However, cluster formation should take into account VANET challenges mentioned earlier in this chapter. Cluster formation in [19] adopts passive clustering to reduce control overhead [16]. It classifies nodes based on "speed groups" [19] that increase the lifetime of a CM in a cluster. On the other hand, a position based clustering technique is introduced in [20] based on node priorities. Location 
information is used to estimate the trip length of each node. Nodes with lower speeds and longer trips have higher priority and thus higher potential to become CHs. A multi-channel MAC protocol is proposed in [21], which supports both safety and comfort applications and allocates a channel for each of the approaches. A cluster in [21] is formed from vehicles moving with similar speeds and moving patterns. Clustering in [22], CASCADE, aims to provide vehicles with an accurate view of the traffic condition ahead. The local view of each node consists of the nodes that lie within its transmission range. CASCADE allows this to extend using a packet received from the $\mathrm{CH}$, which consists of aggregated local views of other nodes in the cluster.

\subsection{Message Dissemination in VANETs}

Routing protocols for vehicular networks aim to provide on-road connectivity between vehicles via efficient IVC or Internet access to vehicles via reliable V2I communications. Because of the availability of the Global Positioning Service (GPS) in most vehicles, most routing techniques used for VANETs are position based routing [23]. Some of position based protocols are presented in [23]. The application oriented nature of VANETs suggests different routing protocols for urban and freeway scenarios. Examples for urban city routing protocols include anchor-based street and traffic aware routing (A-STAR) and geographic source routing (GSR). An example for freeway routing protocols is the greedy perimeter stateless routing (GPSR).

In VANETs, most applications broadcast messages when nodes need to know the situation of other vehicles, or in order to inform other vehicles of urgent situations ahead. A 
broadcasting mechanism is defined by the method of choosing the next node that will rebroadcast the message, so called relay node. Broadcasting techniques in MANETs have been studied in [24], and have been categorized according to the forwarding method into the following five groups,

- Simple flooding: every receiving node rebroadcasts;

- Probability based methods: a receiving node rebroadcasts according to a predetermined probability;

- Area based methods: a receiving node will rebroadcast if it will cover a new sufficient coverage area;

- Neighbor knowledge methods: a receiving node will rebroadcast based on certain neighboring information.

Because of a large number of nodes in VANETs, flooding is not an efficient method. Thus, rebroadcasting should be minimized as much as possible to avoid multiple copies, collisions and retransmissions in order to increase bandwidth utilization but, at the same time, ensure the reception of message to every node in ZOR with a minimum delay. For a two-way highway collision warning shown in Figure 3, a position based broadcast routing protocol called inter-vehicle geocast (IVG) is proposed [25], in which ZOR is defined to be vehicles behind the accident in the same driving direction of accident, and in front of the crashed vehicle in the opposite driving direction. IVG uses the concept of a dynamic relay node which needs to wait for a defer time in every stage before rebroadcasting. The time 
threshold reduces for further nodes, which makes nodes wait before relaying message which might not suit hard delay constraints of CCW.

One method to reduce broadcast redundancy is cluster based broadcasting, where only gateways and $\mathrm{CHs}$ rebroadcast messages in order to improve scalability of the routing protocol and reduce packet collisions. This concept is sometimes used for location discovery process [26]. Cluster based broadcasting is used in BROADCOMM with enhanced message prioritization done by $\mathrm{CH}$ [27]. When a $\mathrm{CH}$ has more than one message to forward, it decides which message to be sent first. The cluster formation in BROADCOMM is geographical based, which divides the highway into equal cells with length equal to the transmission range. In BROADCOMM, the $\mathrm{CH}$ is chosen to be the one closest to the geographical center of the cluster. Clustering in VANETs should consider the high mobility of vehicles and aim to produce stable clusters.

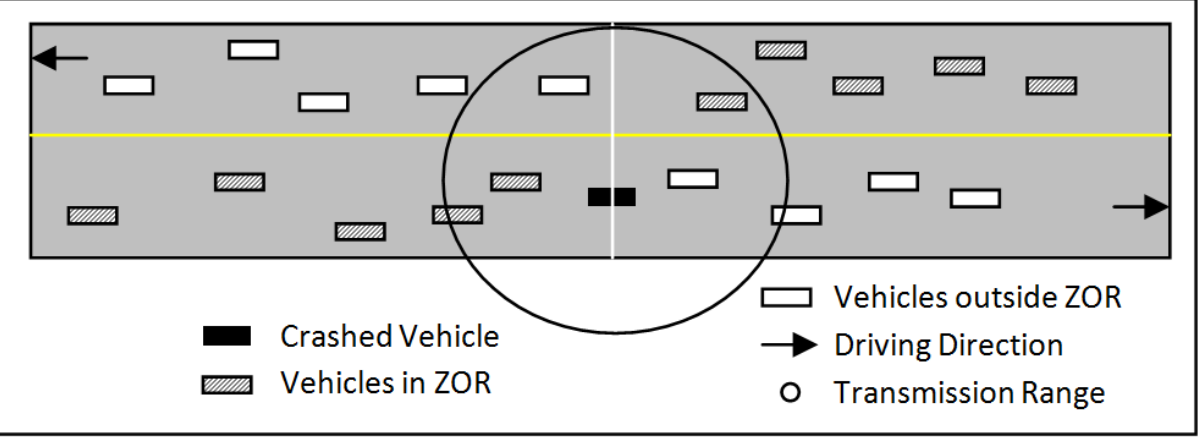

Figure 3. Collision warning broadcast in bidirectional highway using IVG protocol [25] 


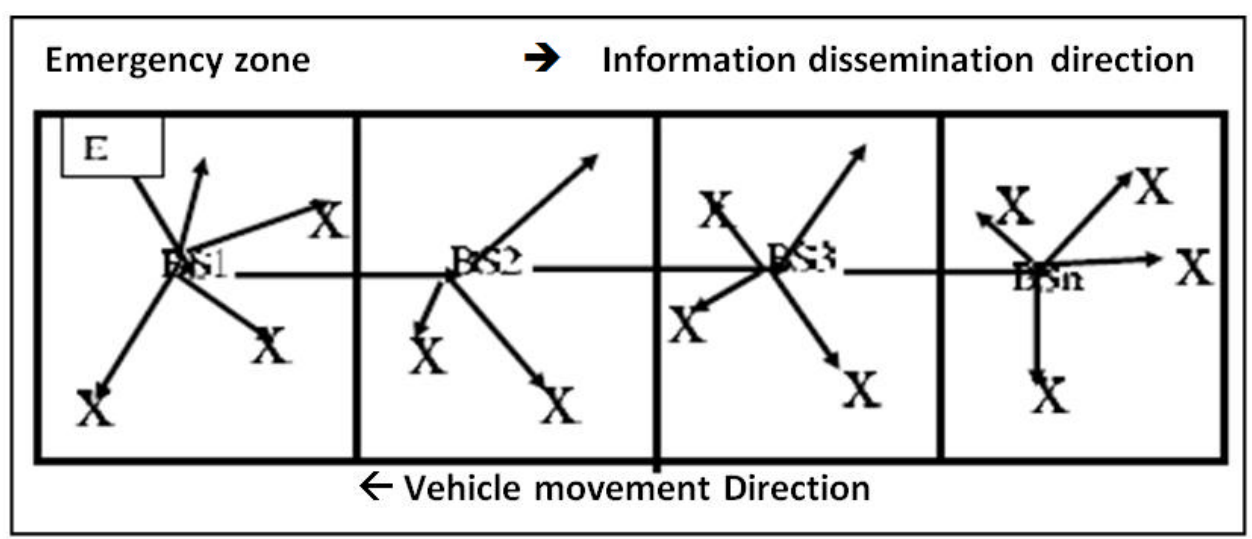

Figure 4. Emergency message dissemination in BROADCOMM [27], with the highway being partitioned into equal sized clusters

\subsection{The Communication Channel}

Dedicated Short Range Communication (DSRC) is a specifically dedicated frequency spectrum for VANETs. The Federal Communications Commission (FCC) has allocated $75 \mathrm{MHz}$ of spectrum for DSRC in the $5.9 \mathrm{GHz}$ band in the US to be used by ITS for both V2V and V2I communications. DSRC system works upon a modified version of IEEE 802.11 [28], which is of class "p" and called Wireless Access in Vehicular Environment (WAVE). WAVE was developed because of the connection establishment delays in IEEE802.11 MAC that follows the three-way transmission (RTS/CTS/Data/ACK). This type of connection delays is not appropriate for VANET application and hence IEEE802.11p has enhancements that supports ITS. The DSRC has seven $10 \mathrm{MHz}$ channels, one channel being a control channel $(\mathrm{CCH})$ restricted for the use of safety transmissions only, while the rest being service channels (SCHs) and can be used for both safety and non safety transmissions, as shown in Figure 5. 


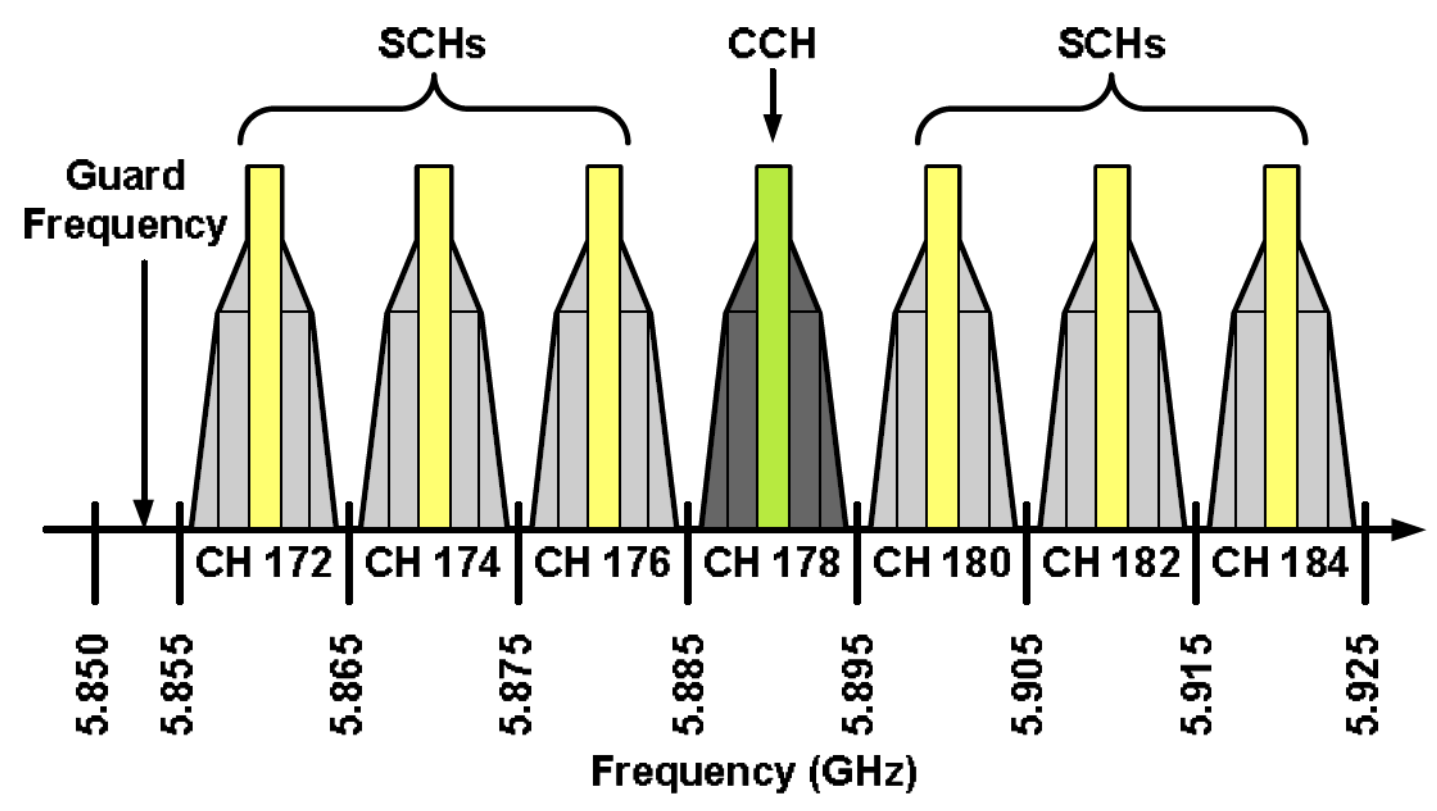

Figure 5. Layout of the U.S. 5.9GHz ITS frequency band [6] 


\section{Chapter 3}

\section{Overview of Traffic Flow Theories}

This chapter provides an overview of the traffic flow theories established in civil engineering. We start by defining some terminologies and characteristics that identify a traffic flow. After that we describe in details the mathematical models characterizing traffic flow for different traffic conditions. This background establishes the base for this research as it is adopted to describe the movement of vehicles in the network.

\subsection{Traffic Flow Conditions and Characteristics}

Civil engineering research has contributed widely in fundamentals of traffic flow theories. It develops mathematical models that describe the behavior of vehicle traffic under different scenarios. The scenarios include vehicle passing (maneuvering), group vehicle travelling (platooning), car following, free-driving, shortest-path-based trip planning, signal stopping or intersection passing. Civil engineering studies also include traffic flow modeling for different types of roads in term of freeway, highway, single lane, multiple lane, or urban city roads. Traffic flow analytical techniques are provided in [9] and proven by measured data collected from real traffic scenarios under real conditions.

Before concluding to the models developed in [9], it is important to define traffic flow characteristics that will be modeled and the parameters and terms used in the modeling. 


\subsubsection{Design Parameters}

One of the important parameters that define the traffic flow condition is the traffic flow rate (or traffic volume) which is the number of vehicles passing a certain point in a given period of time, usually expressed as hourly flow rate. The hourly flow rate is assumed to be known for the designer and thus need not to be calculated, which is the reason behind calling it design flow rate (DFR). Another important parameter that should be introduced is the capacity of a given roadway, $c$, which is defined as "the maximum hourly rate at which persons or vehicles can reasonably be expected to traverse a point or uniform section of a lane or roadway during a given time period under prevailing roadway, traffic, and control conditions" [9]. For uninterrupted traffic applications, such as a freeway with no on-ramps or off-ramps, and under ideal traffic conditions, the capacity $c_{j}$ of freeways with design speeds of 60 or 70 mile per hour (mph) is 2000 vehicles per hour per lane (veh/hr/lane). The ideal conditions represent 12 -foot lanes with adequate lateral clearances with buses, trucks and recreational vehicles excluded from the traffic stream assuming weekday or commuter traffic. However, when ideal cases are not satisfied, the capacity reduces to

$$
c=c_{j} N f_{w} f_{H V} f_{p}
$$

where $N$ is the number of lanes of the freeway, and $f_{w}, f_{H V}$ and $f_{p}$, represent the per lane width, heavy vehicle and driver population factors, respectively. Given the traffic volume and the design capacity of a highway, one can calculate the ratio of the traffic volume to the capacity, the corresponding traffic flow condition, and the level of quality of service provided for the vehicles on road. The limits will be used in classifying which mathematical 
model for traffic characteristics to be used for given design parameters as listed in Table 1 [9].

Another way to identify the flow conditions is from a given traffic density $D$, which is known as the number of vehicles occupying a length of the roadway and is equal to the arrival rate $\lambda_{s}$ in terms of distance. There are some techniques used for calculating the density, such as photography techniques, input-output counts, speed-flow calculations, and occupancy measurements. The speed-flow calculation is given by

$$
D=\frac{V}{\bar{v}}
$$

where $V$ is the traffic volume measured in vehicle per hour (veh/hr) and the density is measured in vehicle per mile per lane (veh/ml/lane), and $\bar{v}$ represents the space mean speed in mph. The traffic flow conditions corresponding to each density level are classified in Table 2 [9].

Table 1. Levels of service for basic freeway sections under ideal conditions and 70mpr design speed [9]

\begin{tabular}{|c|c|c|c|c|c|}
\hline $\begin{array}{c}\text { Level of } \\
\text { service }\end{array}$ & Flow condition & v/c limit & $\begin{array}{c}\text { Service Volume } \\
\text { (veh/hr) }\end{array}$ & $\begin{array}{c}\text { Speed } \\
(\mathbf{m p h})\end{array}$ & $\begin{array}{c}\text { Density } \\
(\mathbf{v e h} / \mathbf{m i l e} / \text { lane })\end{array}$ \\
\hline A & Free & 0.35 & 700 & $\geq 60$ & $\leq 12$ \\
\hline B & Stable & 0.54 & 1100 & $\geq 57$ & $\leq 20$ \\
\hline C & Stable & 0.77 & 1550 & $\geq 54$ & $\leq 30$ \\
\hline D & High Density & 0.93 & 1850 & $\geq 46$ & $\leq 40$ \\
\hline E & Near Capacity & 1.00 & 2000 & $\geq 30$ & $\leq 67$ \\
\hline F & Breakdown & unstable & unstable & $<30$ & $>67$ \\
\hline
\end{tabular}


Table 2. Traffic flow state for different densities [9]

\begin{tabular}{|c|c|c|c|}
\hline $\begin{array}{c}\text { Density } \\
\text { (veh/mile/lane) }\end{array}$ & $\begin{array}{l}\text { Level of } \\
\text { Service }\end{array}$ & \multicolumn{2}{|c|}{ Flow conditions } \\
\hline $0-12$ & $\mathrm{~A}$ & Free-flow operations & \multirow{4}{*}{ Uncongested flow conditions } \\
\hline $12-20$ & B & Reasonable free-flow operations & \\
\hline $20-30$ & $\mathrm{C}$ & Stable operations & \\
\hline $30-42$ & $\mathrm{D}$ & Borders on unstable operations & \\
\hline $42-67$ & $\mathrm{E}$ & Extremely unstable flow operations & Near-capacity flow conditions \\
\hline $67-100$ & \multirow[t]{2}{*}{$\bar{F}$} & Forced or breakdown operations & \multirow[t]{2}{*}{ Congested flow conditions } \\
\hline$>100$ & & Incident situation operations & \\
\hline
\end{tabular}

\subsubsection{Traffic Flow Characteristics}

One of the elements that characterize a traffic flow is the elapsed time of the passage of identical points on two consecutive vehicles, which is called time headway $T$. Time headway is also the combination of the occupancy time for the physical vehicle to pass the observation point and the time gap between the rear of the lead vehicle and the front of the following vehicle. Hence the traffic volume is related to the average time headway $\bar{T}$ in second by

$$
V=\frac{3600}{\bar{T}}
$$

Following the same terminology, distance headway $S$ is a major characteristic that defines a traffic flow. Distance headway is the distance between identical points on two consecutive vehicles and it represents the addition of the lead vehicle's length together with the distance 
gap between the rear of the leading vehicle and the front of the following vehicle. Similar to the time headway, the traffic density is related to the average distance headway $\bar{S}$ in feet by

$$
D=\frac{5280}{\bar{S}} .
$$

Another traffic characteristic is the traffic flow, which represents the traffic load on the roadway and the interaction between these loadings. The capacity described earlier helps in determining the performance of the current traffic load. The traffic flow should not be confused with the parameter Traffic Volume or Traffic Flow Rate, described earlier which represent the average of the traffic flow, while the latter is a random variable and changes with temporal, special, and modal variations of the vehicle flow.

\subsection{Traffic Flow Models}

The traffic flow models developed in [9] are summarized in this subsection. The results in [9] show that the traffic flow characteristic model depends highly on the traffic flow condition, due to the restriction invoked by the level of interaction between nearby vehicles. For example, for a very low traffic density, the interaction between vehicles is almost negligible. As a result, vehicles move independently and enjoy a maximum level of service (i.e., service level A or B as given in Table 1). As the traffic flow density increases, the interactions between vehicles increase, eliminating the free flow state. Finally when the traffic flow density approaches the road capacity, the interactions further increase, which eliminates randomness in the movement of vehicles and leads to almost steady movement with deterministic traffic characteristic values governed by car following models. 


\subsubsection{Low Density}

For low traffic density, the interactions between vehicles are very low and almost negligible. Thus, it is realistic to assume completely independent movement from vehicle to vehicle. It is found that the arrival process of vehicles to a highway can be modeled as a Poisson process with an arrival rate $\lambda_{t}[9]$. Hence, the probability that $n$ vehicles arrive in a time period $t$ can be calculated using

$$
P(X=n)=\frac{\left(x \lambda_{t}\right)^{n}}{n !} e^{-\lambda_{t} x}
$$

where $X$ is the number of vehicles arriving to a highway in a time interval $x$. As for the time headway distribution, it is described in [9] as an exponential distribution of parameter $\left(\frac{1}{\lambda_{t}}\right)$ with probability density function (PDF) $f_{T}(t)=\lambda_{t} e^{-\lambda_{t} t}, t \geq 0$. The PDF allows the time headway to go to zero and actually give it the highest relative likelihood of occurrence, which is not realistic. To avoid this drawback, the time is shifted with the minimum allowable time headway $\tau$, resulting in the PDF given by

$$
f_{T}(t)= \begin{cases}\lambda_{t} e^{-\lambda_{t}(t-\tau)}, & t \geq \tau \\ 0, & t<\tau\end{cases}
$$

The velocity of the vehicles moving in a low density is assumed to be constant on average. Hence, the distance headway distribution can follow the same distribution as that for the time headway and thus be shifted exponential with parameter $\left(\frac{1}{\lambda_{s}}\right)$ with PDF

$$
f_{S}(s)= \begin{cases}\lambda_{s} e^{-\lambda_{s}(s-\alpha)}, & s \geq \alpha \\ 0, & s<\alpha\end{cases}
$$


where $\alpha$ here corresponds to the minimum allowable distance for the vehicle movements to be independent. The average distance headway is $\bar{S}=\frac{1}{\lambda_{s}}+\alpha$.

\subsubsection{High Density}

When the traffic volume exceeds the road capacity and roadway becomes congested, the traffic flow rate becomes almost constant everywhere. Hence, the traffic flow can be calculated by multiplying the traffic volume by the time. As a matter of fact all traffic characteristics are approximately constant when the traffic is congested, yet it is suggested to model the time headway with a truncated normal probability distribution in order to account for driver errors while attempting to keep a constant time headway [9]. Thus, the PDF of the time headway for high dense traffic is given by

$$
f_{T}(T=t)=\frac{1}{\sqrt{2 \pi} \sigma \gamma} e^{-\frac{(t-\bar{T})^{2}}{2 \sigma^{2}}}, \mathrm{t} \geq \tau
$$

Given a certain $V$ value, the mean time headway can be calculated. The standard deviation $\sigma$ can be calculated from the minimum time headway $\tau$ by

$$
\sigma=\frac{\bar{T}-\tau}{2}
$$

Since $T$ is lower bounded by $\tau$, the normalization factor $\gamma$ is equal to $1-\Phi\left(\frac{\tau-\bar{T}}{\sigma}\right)$, where $\Phi($. is the cumulative distribution function $(\mathrm{CDF})$ of the standard normal distribution. For the distance headway, it is clear that, in a congested traffic flow, vehicle movement is constrained by the movement of the lead vehicle, so that the following vehicle attempts to maintain a safe distance according to car following models [9]. This is done when the 
following vehicle accelerates or decelerates according to the changes of speed of the lead vehicle. However, the velocity of an individual vehicle velocity is random and difficult to model. Although velocity change models are presented for many scenarios (e.g. car maneuver, passing intersection, stopping on a traffic light, etc) [9], it is not clear how often each of the scenarios occurs for a vehicle during its trip on a highway.

\subsubsection{Intermediate Density}

The intermediate state is the most difficult to analyze since it cannot be considered as pure random or pure deterministic (constant). In this case, some vehicles tend to drive freely with a high QoS level, while others tend to drive in platoons governed by car following models. For simplicity, we will assume that the arrival process is Poisson as given in (3.5). To find the probability distribution of the time headway, a generalized model is suggested in [9] based on Pearson type III probability distribution and a composite model which combines platoons and free driving. Let the fraction of vehicles traveling in platoons be $P_{P}$ and the fraction of vehicles driving not in platoons be $P_{N P}$. We have $\mathrm{P}_{\mathrm{P}}+\mathrm{P}_{\mathrm{NP}}=1$. The PDF of the time headway when the traffic flow is in the intermediate zone is then

$$
f_{T}(T)=P_{P} \frac{1}{\sqrt{2 \pi} s_{p}} e^{-\frac{\left(t-\mu_{p}\right)^{2}}{2 s p^{2}}}+P_{N P} \lambda_{N P} e^{-\lambda_{N P}(t-\tau)}
$$

where $\bar{T}_{N P}$ is the average time headway for vehicles travelling not in platoons, $\mu_{p}$ is the average time headway for vehicles travelling in platoons, $s_{N P}$ and $s_{p}$ are the standard deviation for vehicles not in platoons and those travelling in platoons, respectively. To 
calculate parameters $P_{P}, \lambda_{N P}$, and $\bar{T}$ three independent parameters $\mu_{p}, \sigma$ and $\tau$ should be determined first by inspection. Then the following relations can be used

$$
\begin{aligned}
& P_{P}=\frac{\mu_{p}}{\bar{T}} \\
& \lambda_{N P}=\frac{1}{\bar{T}_{N P}-\tau}=\frac{1}{s_{N P}} \\
& \bar{T}=P_{P} \mu_{p}+P_{N P} \bar{T}_{N p}
\end{aligned}
$$

Table 3 summarizes the mathematical modeling. In this work, we assume that the velocity is normally distributed for high and intermediate traffic flow conditions, with a range of allowable values for each flow condition. Since vehicles enjoy a maximum level of service in a low traffic density, we assume that vehicles drive with a constant velocity equal to the maximum allowable velocity for the highway. 


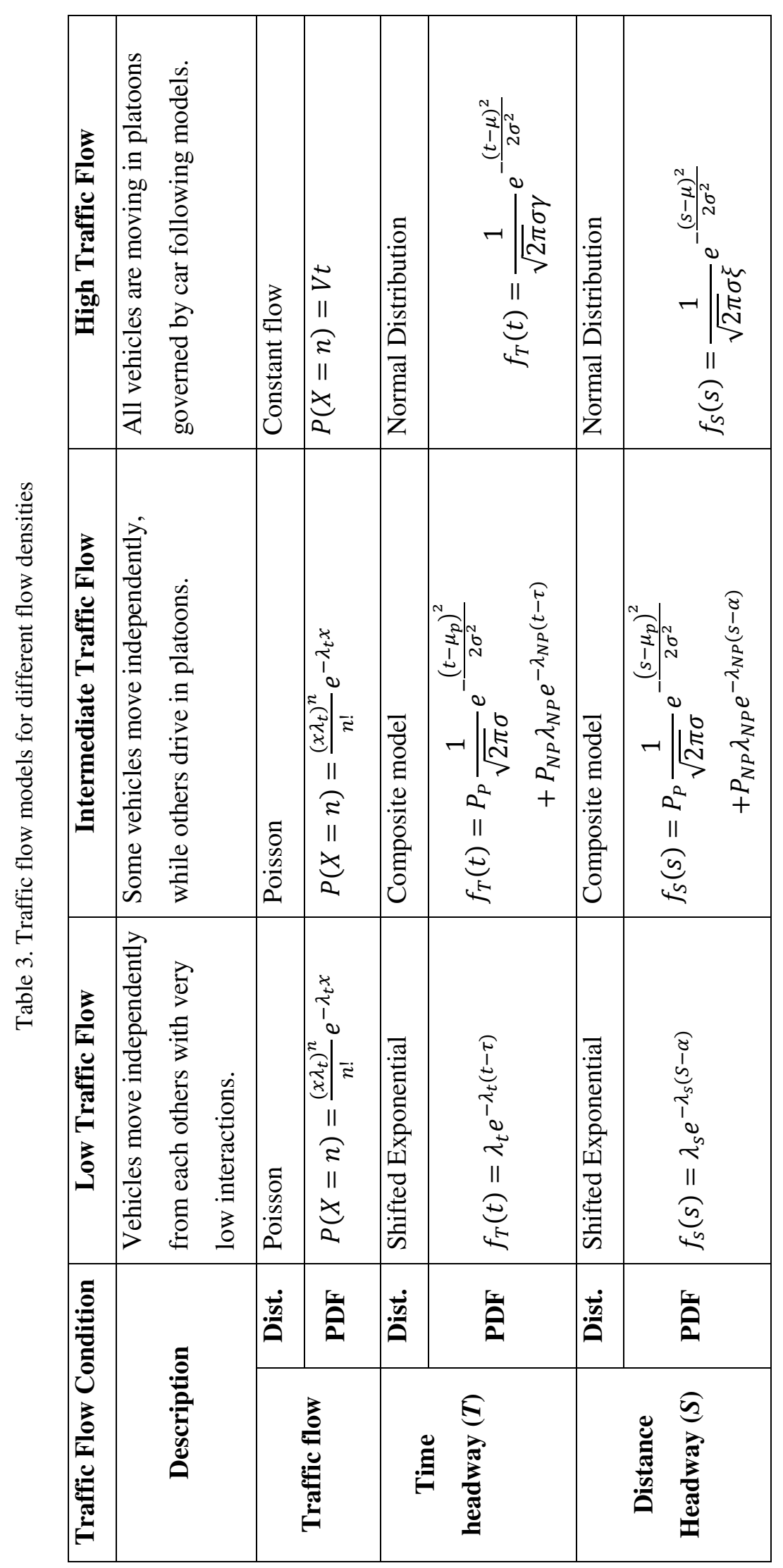




\section{Chapter 4}

\section{Modeling and Analysis for Cluster based Emergency}

\section{Broadcasting Delay}

This chapter presents a novel approach to investigate the broadcasting delay for emergency message dissemination and provides mathematical models for it. The broadcasting scheme under investigation is cluster based, where only the backbone of the clustered infrastructure, consisting of $\mathrm{CHs}$ and gateways, is responsible of relaying the safety messages. The clustered infrastructure is assumed to be already existing when an emergency happens and is already in use by some other application, such as medium access control (MAC), scheduling, or providing scalability. The vehicle movement is governed by the mathematical models described in Chapter 3. This chapter is organized as follows: in section 4.1, the system model under study and assumptions made for this research are described, the models considered for the movement of vehicles are then summarized, and finally the clustered infrastructure assumed is described. Section 4.2 provides detailed description of the broadcasting procedure, while section 4.3 discusses the models for the three traffic flow states. Finally, section 4.4 presents numerical results and analysis supported with simulation results and discussions. 


\subsection{System Model}

Consider a VANET for a three-lane highway in one direction. For uninterrupted traffic applications, such as a freeway with no on-ramps or off-ramps, it is reasonable to assume ideal conditions and hence the capacity of highways with design speeds of $60-70$ $\mathrm{mph}$ is 2000 vehicles per mile per lane (veh/ml/lane). Vehicles enter the system according to a Poisson process in terms of distance with an arrival rate $\lambda_{s}$ equal to the traffic flow density. We assume that the traffic flow density is split equally among the lanes and that the traffic flow model is not affected by the incident during message broadcasting. The density determines the traffic flow state and the speed limits according to Table 1 [9]. We assume that the subsection of highway is far enough from any on-ramps or off-ramps so that the speeds of the vehicles are in a steady state.

We consider all ranges of traffic flow densities representing sparse, intermediate, and congested traffic conditions as described in details in section 4.2. All vehicles are equipped with GPS receivers and with same radio capabilities. As described in Chapter 2, IVC is allowed through a single channel, one of the seven channels specified in the DSRC. The DSRC channel supports a high data rate of 6-27 Mbps with a transmission range up to 3280 feet (ft). Normally, safety messages require only a low bandwidth and have a size of hundreds of bytes (e.g. 200-500 bytes). Figure 6 illustrates the VANET under consideration, where the message dissemination direction opposes the traffic flow direction. 


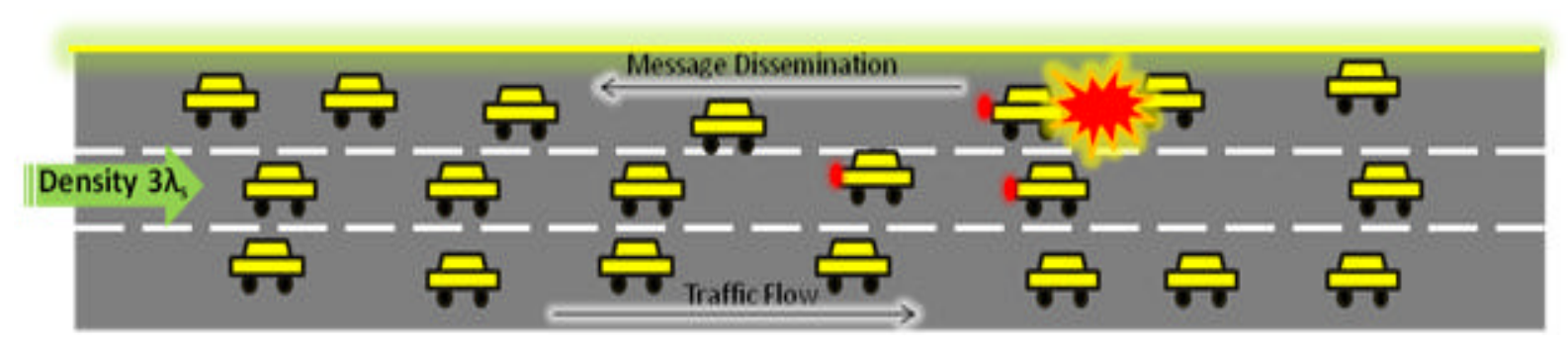

Figure 6. An illustration of the VANET under consideration

\subsubsection{Vehicle Mobility Models}

In this work, we are more interested in the distance headway distribution, and the spatial distribution of vehicles along the road other than their time distribution. For a low traffic density, since the vehicles enjoy maximum level of service and maximum speed limits, it is reasonable to assume that vehicles move at constant velocity and do not interact with each other. Hence, the arrival process of vehicles to the highway can be modeled as a Poisson process with an arrival rate $\left(3 \lambda_{s}\right)$, which is equal to the traffic density, and is split equally among the lanes. Assuming that each lane is modeled similarly to the combined highway, the probability that there are $n$ vehicles in a road section of length $x$ per lane is given by

$$
P(N=n)=\frac{\left(x \lambda_{s}\right)^{n}}{n !} e^{-\lambda_{s} x} .
$$

The inter-vehicle spacing for a low traffic density, as mentioned in Chapter 3, follows exponential distribution with a PDF given by (3.7). As for a dense highway, approximately constant distance between adjacent vehicles is assumed [9]. Inspired from the time headway distribution for high density given in (3.8) and discussed in Chapter 3, we model the distance 
headway by a truncated normal distribution, where the randomness accounts for the driving error in keeping a constant distance headway. The PDF of $S$ in a high traffic density is then given by

$$
f_{S}(s)=\frac{1}{\sqrt{2 \pi} \sigma \xi} e^{-\frac{(s-\mu)^{2}}{2 \sigma^{2}}}, s \geq \alpha
$$

where $\mu$ is the mean distance headway, which is the reciprocal of the density, $\alpha$ is the minimum distance headway allowed for safety reasons, and $\sigma$ is the standard deviation and calculated from $\sigma=\frac{\mu-\alpha}{2}$. Since $S$ is lower bounded by $\alpha$, the constant $\xi$ in the truncated normal distribution is equal to $1-\Phi\left(\frac{\alpha-\mu}{\sigma}\right)$ where $\Phi($.$) is the CDF of the standard normal$ distribution. For intermediate traffic flow densities, we use the composite distribution model as discussed in Chapter 3 and (3.14) is re-written as

$$
f_{S}(s)=P_{P} \frac{1}{\sqrt{2 \pi} \sigma} e^{-\frac{(s-\mu)^{2}}{2 \sigma^{2}}}+P_{N P} \lambda_{s} e^{-\lambda_{S}(s-\alpha)},
$$

where the fraction $P_{P}$ can be considered as the ratio of the highway traffic density to the density threshold $D_{p}$ above which all vehicles are assumed to be in platoons [9] .

\subsubsection{Cluster infrastructure}

For a VANET with a large number of vehicles (nodes), node clustering is an efficient approach to achieve scalability and stability in network control and operation. Here, we assume that node clustering is in place for other network services and applications, and explore the clustered network infrastructure for disseminating emergency messages. There 


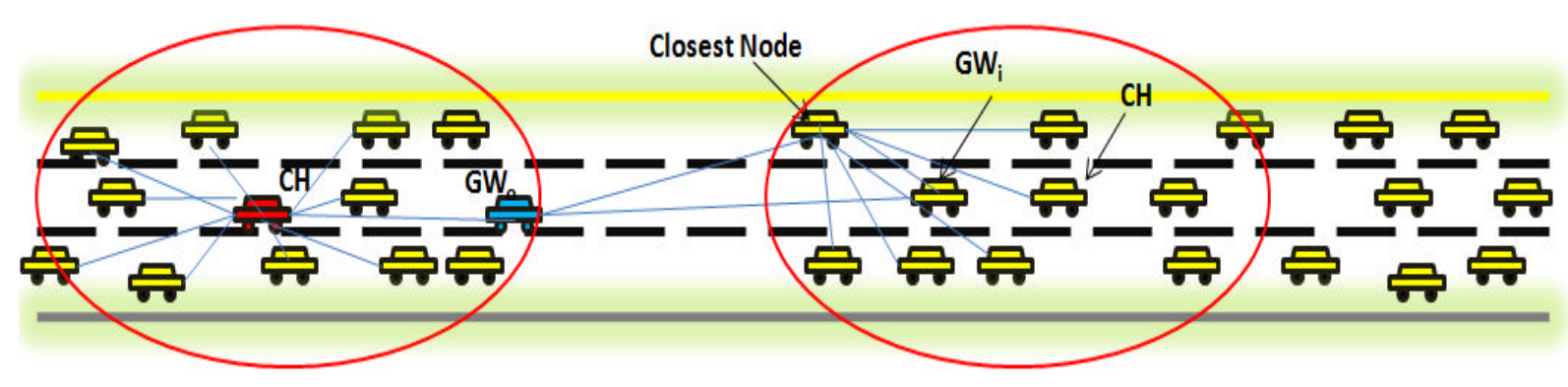

Figure 7. Cluster formation process and gateways $\left(\mathrm{GW}_{\mathrm{i}}\right.$ and $\left.\mathrm{GW}_{\mathrm{o}}\right)$ selection

are different ways of node clustering as shown in Figure 2. To minimize the number of clusters that covers the highway, we choose to have non-overlapping clusters. Nonoverlapping clusters, shown in Figure 2 (a), fit the nature of VANETs which makes overlapping clusters impossible because of some empty sections.

Vehicles on highway are initially clustered by a cluster formation request (CFR) message from an arbitrary node. Since middle lane vehicles have the least relative speed on average with vehicles on the other lanes, $\mathrm{CHs}$ are chosen from middle lane nodes. Hence, only middle lane receivers of the CFR message compete for the $\mathrm{CH}$ position, and the source of CFR message announces the $\mathrm{CH}$ winner based on the first reply received. The borders of a cluster is limited by the transmission range of the $\mathrm{CH}$, creating single-hop non-overlapping clusters as shown in Figure 2 (a). Thus CMs are those nodes that lie within the transmission range of the $\mathrm{CH}$.

Communication between non-overlapping clusters requires gateways, which are elected from nodes closest to the cluster borders and preferably on the middle lane. A gateway selection procedure is described in [29]. By avoiding cluster overlapping and 
maximizing the coverage area of a cluster, the required number of clusters is minimized. The winner gateway $\left(\mathrm{GW}_{\mathrm{o}}\right)$ selects a gateway $\left(\mathrm{GW}_{\mathrm{i}}\right)$ for the next cluster, and the newly selected gateway $\left(\mathrm{GW}_{\mathrm{i}}\right)$ chooses the most distant node within its transmission range to be the $\mathrm{CH}$ of the next cluster, as illustrated in Figure 7. This process repeats until the whole highway is clustered. It is obvious that the clusters move with the movement of $\mathrm{CHs}$. Each $\mathrm{CH}$ keeps a table list of all its CMs, while CMs can leave and enter a cluster without imposing any reclustering process.

\subsection{Cluster Broadcast Procedure}

A fully clustered VANET is shown in Figure 8, where node A represents a source node that initiates an emergency message to be transmitted to every node in the ZOR in a direction opposing the traffic flow as shown in Figure 3. Node B represents the last vehicle in the ZOR. Initially, to calculate the total broadcasting delay $T_{D}$, we assume that the network is static. Hence, the total delay $T_{D S}$ for a message broadcasted from source A to reach node B with a static infrastructure is given by

$$
T_{D S}=T_{1 s t}+N_{C} T_{C H}+\left(N_{C}-1\right)\left(T_{G W o}+T_{G W i}\right)
$$

where $N_{C}$ is the number of clusters in ZOR, $T_{1 s t}$ is the broadcast transmission delay of safety message from the source node to its $\mathrm{CH}, T_{C H}$ is the $\mathrm{CH}$ broadcast delay to its $\mathrm{CM}, T_{G W o}$ and $T_{G W i}$ are the delays of the inter-cluster broadcast from gateway $\mathrm{GW}_{\mathrm{o}}$ to gateway $\mathrm{GW}_{\mathrm{i}}$ and the intra-cluster broadcast from $\mathrm{GW}_{\mathrm{i}}$ to its $\mathrm{CH}$, respectively. The calculation of $T_{D}$ in (4.4) shows clearly how an increase in the cluster size reduces the total delay by reducing 


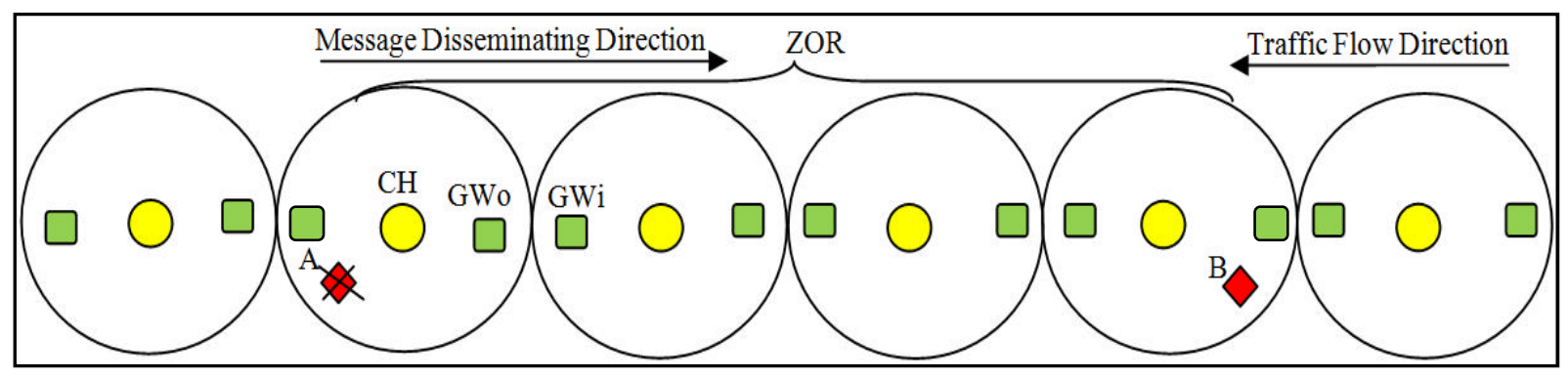

Figure 8. Emergency broadcasting from a source node A through the backbone of the clustered infrastructure till the last node in ZOR

the number of clusters. This calculation is based on a static network infrastructure which is not suitable for highly dynamic VANETs. To make the calculations more practical, it is important to consider mobility and random behaviors of vehicles on road. Figure 9 shows a flow chart of the proposed broadcasting procedure which consists of three stages.

In the first stage, the safety message is with the $\mathrm{CH}$, which will rebroadcast the message directly if its gateway $\mathrm{GW}_{\mathrm{o}}$ is available, this event is denoted by $G_{A}$; otherwise denoted by $G_{N A}$, a gateway re-election process is initiated with a total time of $T_{E}$. Note that a $\mathrm{CH}$ will broadcast directly after gateway re-election, since checking step is not required for a newly elected gateway. Thus, the average $\mathrm{CH}$ broadcast delay is

$$
\begin{aligned}
E\left[T_{C H}\right] & =E\left[T_{C H} \mid G_{A}\right] P\left(G_{A}\right)+E\left[T_{C H} \mid G_{N A}\right] P\left(G_{N A}\right) \\
& =T_{C H} P\left(G_{A}\right)+\left(T_{C H}+T_{E}\right) P\left(G_{N A}\right),
\end{aligned}
$$

where $\mathrm{P}(\mathrm{x})$ denotes the probability of an event $\mathrm{x}$.After the $\mathrm{CH}$ broadcasts, the gateway $\mathrm{GW}_{\mathrm{o}}$ has a copy of the safety message and the second stage begins. 


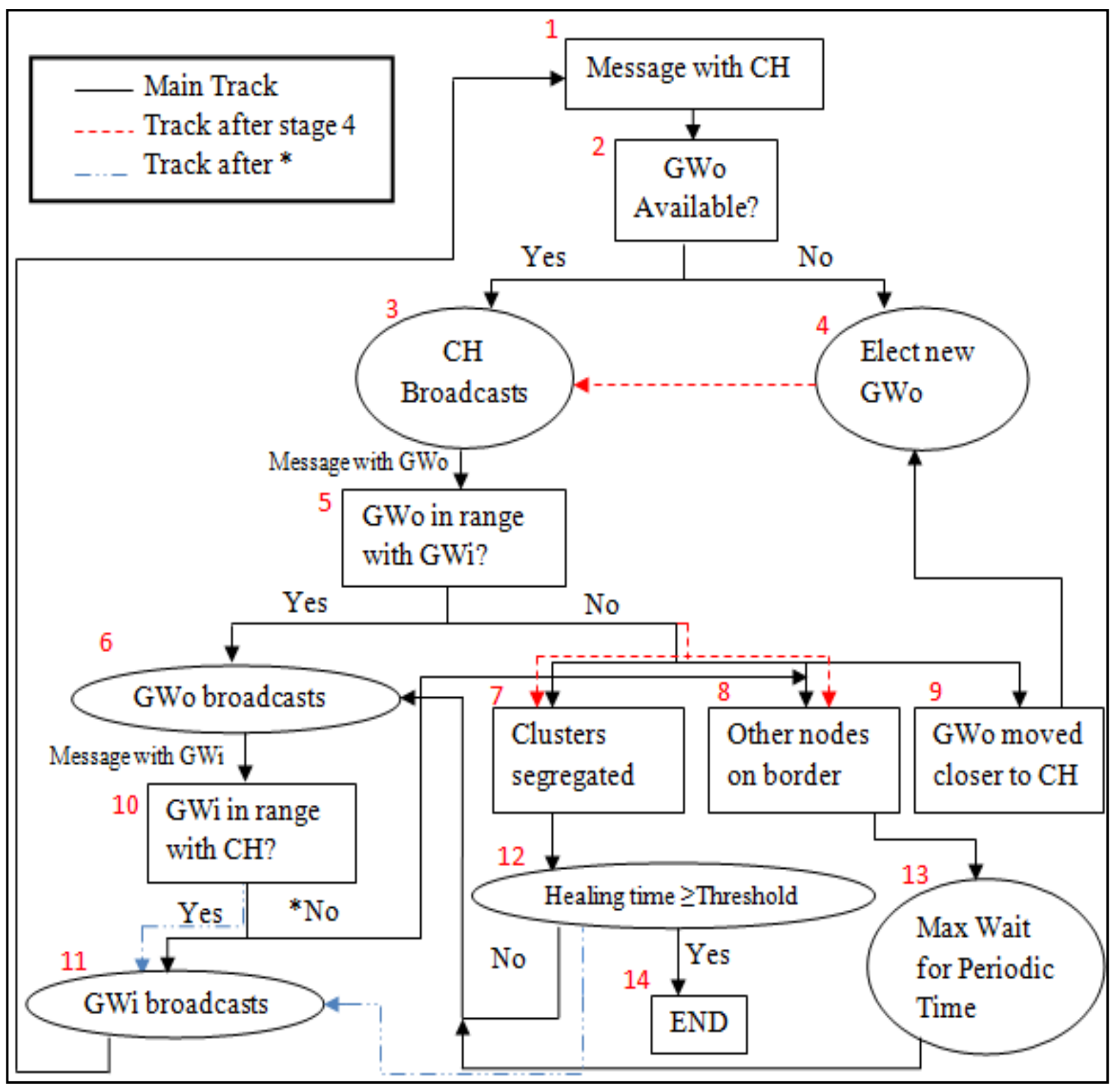

Figure 9. Mobility aware cluster-based broadcasting procedure

Before rebroadcasting the message, the gateway $\mathrm{GW}_{\mathrm{o}}$ checks for its connection with the gateway $\left(\mathrm{GW}_{\mathrm{i}}\right)$ of the next cluster. The connection is denoted by $C_{o, i}$. Given that $\mathrm{GW}_{\mathrm{o}}$ is disconnected from $\mathrm{GW}_{\mathrm{i}}$, an event denoted by $D_{o, i}$, one of the following three events occurs: 
1) Event $M_{o}-\mathrm{GW}_{\mathrm{o}}$ has moved closer to its $\mathrm{CH}$;

2) Event $M_{i}-\mathrm{GW}_{\mathrm{i}}$ has moved closer to its $\mathrm{CH}$;

3) Event $C_{g}$ - The two clusters are segregated.

Re-election of the gateway $\mathrm{GW}_{\mathrm{o}}$ as in stage one will solve the problem in case 1). In case 2), the gateway $\mathrm{GW}_{\mathrm{o}}$ needs to periodically check for the gateway $\mathrm{GW}_{\mathrm{i}}$ and waits for a maximal period of the cluster maintenance time denoted by $T_{P}$. In case 3 ), the broadcasting halts for a healing time denoted by $T_{H}$. Hence, the average rebroadcasting delay at the gateway $\mathrm{GW}_{\mathrm{o}}$ can be calculated by

$$
\begin{aligned}
E\left[T_{G W o}\right]= & T_{G W o} P\left(C_{o, i}\right)+\left[\left(T_{G W o}+T_{H}\right) P\left(C_{g}\right)+\left(T_{G W o}+T_{E}\right) P\left(M_{o}\right)\right. \\
& \left.+\left(T_{G W o}+T_{P}\right) P\left(M_{i}\right)\right] P\left(D_{o, i}\right) .
\end{aligned}
$$

Since the healing time is dependent on the actual catching up of vehicles to the leading ones [7], an upper bound is used for $T_{H}$ to meet the total latency requirement. When such a threshold is exceeded, implying no need for safety message to disseminate further, the broadcasting stops.

The third stage starts after the safety message arrives at the gateway $\mathrm{GW}_{\mathrm{i}}$. The gateway may be disconnected from its $\mathrm{CH}$ due to relative mobility between them. Hence, gateway $\mathrm{GW}_{\mathrm{i}}$ needs to wait for at most $T_{P}$ before it relays the message to the new gateway which relays it at once to the $\mathrm{CH}$, with a total delay given by

$$
E\left[T_{G W i}\right]=T_{G W i} P\left(G_{A}\right)+\left(2 T_{G W i}+T_{P}\right) P\left(G_{N A}\right) .
$$


Note that it is unlikely to have cluster segregation, since it corresponds to a half empty cluster with only gateway. Hence, this case is neglected in this study. Substituting (4.5)-(4.7) in (4.4), we have an expression of the total broadcasting delay of an emergency message as a function of the number of clusters on a highway. Taking account of the randomness in the mobility, the total broadcasting delay $T_{D R}$ is given by

$$
\begin{aligned}
T_{D R}= & T_{1 s t}+N_{C}\left(T_{C H} P\left(G_{A}\right)+\left(T_{C H}+T_{E}\right) P\left(G_{N A}\right)\right)+ \\
& +\left(N_{C}-1\right)\left(T_{G W o} P\left(C_{o, i}\right)+\left[\left(T_{G W o}+T_{H}\right) P\left(C_{g}\right)\right.\right. \\
& \left.+\left(T_{G W o}+T_{E}\right) P\left(M_{o}\right)+\left(T_{G W o}+T_{P}\right) P\left(M_{i}\right)\right] P\left(D_{o, i}\right) \\
& \left.+T_{G W i} P\left(G_{A}\right)+\left(2 T_{G W i}+T_{P}\right) P\left(G_{N A}\right)\right) .
\end{aligned}
$$

\subsection{Cluster Broadcast Delay Models}

In (4.8), the probability terms depend on the mathematical models of the traffic flow density. Hence, the delay is modeled differently based on the three categories of the traffic flow density. In the case of a high density, it is highly likely that there are always neighboring nodes available for connection due to small distance headways. Hence, the total broadcasting delay for a high traffic density can be approximated by $T_{D S}$. With a low traffic density, the mathematical distributions (3.7) and (4.1) can be used to calculate the probability terms. For example, in the first stage of message broadcasting, the probability of gateway $\mathrm{GW}_{\mathrm{o}}$ being available is the probability that the distance $L$ between the $\mathrm{CH}$ and $\mathrm{GW}_{\mathrm{o}}$ is less than the transmission range $R$. Since the inter-vehicle spacing is exponentially distributed, $L$ follows a gamma distribution with a CDF given by 


$$
F_{l}(d)=1-\sum_{i=0}^{k-1} \frac{\left(\lambda_{S}(L-\alpha)\right)^{i}}{i !} e^{-\lambda_{S}(L-\alpha)},
$$

where $k$ is the number of nodes between $\mathrm{CH}$ and $\mathrm{GW}_{\mathrm{o}}$, with the average of $k$ be $\lambda_{s} R-2$ from (4.1). As for the probability of the event $C_{o, i}$, we can obtain it based on the distance headway distribution (3.7). Equation (4.1) can be used to find the probability of the event $C_{g}$, which corresponds to having an empty road section with length $R$. Finally, the probability that a gateway moves closer to its $\mathrm{CH}$ (i.e., event $M_{o}$ and $M_{i}$ ), with link disconnection, is the probability that it is no longer located at the cluster border. We consider this event happens when the gateway is in the closer half of $R$ to its $\mathrm{CH}$ and calculate the probability based on (4.9). Substituting the probability calculations summarized in Table 4 in (4.8),the total broadcasting delay $T_{D L}$ for a low traffic flow density can be found by

$$
\begin{aligned}
T_{D L}= & T_{1 s t}+N_{C}\left(T_{C H}+T_{E} \sum_{i=0}^{R \lambda_{S}-3} \frac{\left(\lambda_{S}(R-\propto)\right)^{i}}{i !} e^{-\lambda_{S}(R-\alpha)}\right) \\
& +\left(N_{C}-1\right)\left(T_{G W o}\left(1-e^{-\lambda_{S}(R-\alpha)}\right)+\left[\left(T_{G W o}+T_{H}\right) e^{-3 \lambda_{S} R}\right.\right. \\
& \left.+\left(T_{E}+2 T_{G W o}+T_{P}\right)\left(1-\sum_{i=0}^{R \lambda_{S}-3} \frac{\left(\lambda_{S}\left(\frac{R}{2}-\propto\right)\right)^{i}}{i !} e^{-\lambda_{S}\left(\frac{R}{2}-\alpha\right)}\right)\right] e^{-\lambda_{S}(R-\alpha)} \\
& +T_{G W i}\left(1-\sum_{i=0}^{R \lambda_{S}-3} \frac{\left(\lambda_{S}(R-\alpha)\right)^{i}}{i !} e^{-\lambda_{S}(R-\alpha)}\right) \\
& \left.+\left(2 T_{G W i}+T_{P}\right) \sum_{i=0}^{R \lambda_{S}-3} \frac{\left(\lambda_{S}(R-\alpha)\right)^{i}}{i !} e^{-\lambda_{S}(R-\alpha)}\right) .
\end{aligned}
$$

From (4.4) and (4.10), the total broadcasting delay $T_{D I}$ for an intermediate traffic flow density is given by 


$$
T_{D I}=P_{N P} T_{D L}+P_{P} T_{D S}
$$

Table 4. List of the probability values required for modeling

\begin{tabular}{|c|c|c|c|}
\hline $\begin{array}{c}\text { Probabi } \\
\text { lity }\end{array}$ & Low density & $\begin{array}{c}\text { High } \\
\text { density }\end{array}$ & Intermediate Density \\
\hline$P\left(G_{A}\right)$ & $1-\sum_{i=0}^{R \lambda_{S}-3} \frac{\left(\lambda_{S}(R-\propto)\right)^{i}}{i !} e^{-\lambda_{S}(R-\alpha)}$ & 1 & $\begin{array}{l}P_{N P}[1- \\
\left.\sum_{i=0}^{R \lambda_{s}-3} \frac{\left(\lambda_{s}(R-\alpha)\right)^{i}}{i !} e^{-\lambda_{S}(R-\alpha)}\right]+P_{P}\end{array}$ \\
\hline$P\left(G_{N A}\right)$ & $\sum_{i=0}^{R \lambda_{s}-3} \frac{\left(\lambda_{S}(R-\propto)\right)^{i}}{i !} e^{-\lambda_{S}(R-\propto)}$ & 0 & $P_{N P}\left[\sum_{i=0}^{R \lambda_{S}-3} \frac{\left(\lambda_{S}(R-\alpha)\right)^{i}}{i !} e^{-\lambda_{S}(R-\alpha)}\right]$ \\
\hline$P\left(C_{o, i}\right)$ & $1-e^{-\lambda_{S}(R-\alpha)}$ & 1 & $P_{N P}\left[1-e^{-\lambda_{S}(R-\alpha)}\right]+P_{P}$ \\
\hline$P\left(C_{g}\right)$ & $e^{-3 \lambda_{S} R}$ & 0 & $P_{N P} e^{-3 \lambda_{S} R}$ \\
\hline$P\left(M_{o}\right)$ & $1-\sum_{i=0}^{R \lambda_{S}-3} \frac{\left(\lambda_{s}\left(\frac{R}{2}-\propto\right)\right)^{i}}{i !} e^{-\lambda_{s}\left(\frac{R}{2}-\propto\right)}$ & - & $\begin{array}{l}P_{N P}[1- \\
\left.\sum_{i=0}^{R \lambda_{s}-3} \frac{\left(\lambda_{s}\left(\frac{R}{2}-\alpha\right)\right)^{i}}{i !} e^{-\lambda_{S}\left(\frac{R}{2}-\alpha\right)}\right]\end{array}$ \\
\hline$P\left(M_{i}\right)$ & $1-\sum_{i=0}^{R \lambda_{S}-3} \frac{\left(\lambda_{s}\left(\frac{R}{2}-\propto\right)\right)^{i}}{i !} e^{-\lambda_{s}\left(\frac{R}{2}-\propto\right)}$ & - & $\begin{array}{l}P_{N P}[1- \\
\left.\sum_{i=0}^{R \lambda_{S}-3} \frac{\left(\lambda_{s}\left(\frac{R}{2}-\alpha\right)\right)^{i}}{i !} e^{-\lambda_{S}\left(\frac{R}{2}-\alpha\right)}\right]\end{array}$ \\
\hline$P\left(D_{o, i}\right)$ & $e^{-\lambda_{S}(R-\alpha)}$ & 0 & $P_{N P} e^{-\lambda_{S}(R-\alpha)}$ \\
\hline
\end{tabular}

\subsection{Numerical Analysis and Simulation Results}

This section presents numerical analysis and simulation results for the broadcasting delay based on the mathematical model described earlier. We consider an emergency message with a size of 500 bytes for broadcasting to all the vehicles in ZOR of 15.5 miles behind the source vehicle. The message is transmitted at a rate of $6 \mathrm{Mbps}$ with an equal single hop-broadcast transmission delay of $0.666 \mathrm{~ms}$ for $T_{1 s t}, T_{C H}, T_{G W i}$, and $T_{G W o}$ in both simulations 
and analysis. Since a gateway election process [29] involves sending a request and an announcement message, we set $T_{\text {elect }}$ to be $1 \mathrm{~ms}$ and the cluster maintenance time period to be $4 \mathrm{~ms}$. The number of clusters partitioning the ZOR is $N_{c}=\frac{15.5}{2 R}$. In a low traffic density condition, it is possible to have significant inter-cluster spacing, which is assumed to follow a uniform distribution on $[0, \mathrm{R}]$. As a result, $N_{C}$ has a probability mass function of $P_{N_{c}}\left(N_{c}=\right.$ $n)=\frac{15.5}{R n^{2}}$ with an average of $\ln \frac{2}{3}\left(\frac{15.5}{R}\right)$.

For a low density, the distance headway should be kept large enough to guarantee a minimum service level that supports free driving. Hence, $\alpha$ is calculated from the maximum density in the low flow condition region and found to be 132ft. Reference [9] provides detailed investigation for the average time headway under which all vehicles are considered in platoons. The corresponding traffic volume threshold is $2400 \mathrm{veh} / \mathrm{hr}$. Under the assumption that all vehicles in a high traffic flow region move in platoons, the density threshold $D_{p}$ is $67 \mathrm{veh} / \mathrm{ml} / \mathrm{lane}$ from

Table 2. We neglect the cluster segregation since detailed analysis for such a case is provided in [7]. Figure 10 plots the analytical results for total broadcasting delay as a function of the transmission range $R$ for the traffic flow density of 10, 20, 50 and 80 $\mathrm{veh} / \mathrm{ml} / \mathrm{lane}$. As the transmission range increases, the number of clusters in ZOR decreases, resulting in a decrease in the total delay. On the other hand, a lower traffic density results in a larger delay. This is due to more likely disconnections between a $\mathrm{CH}$ and its 


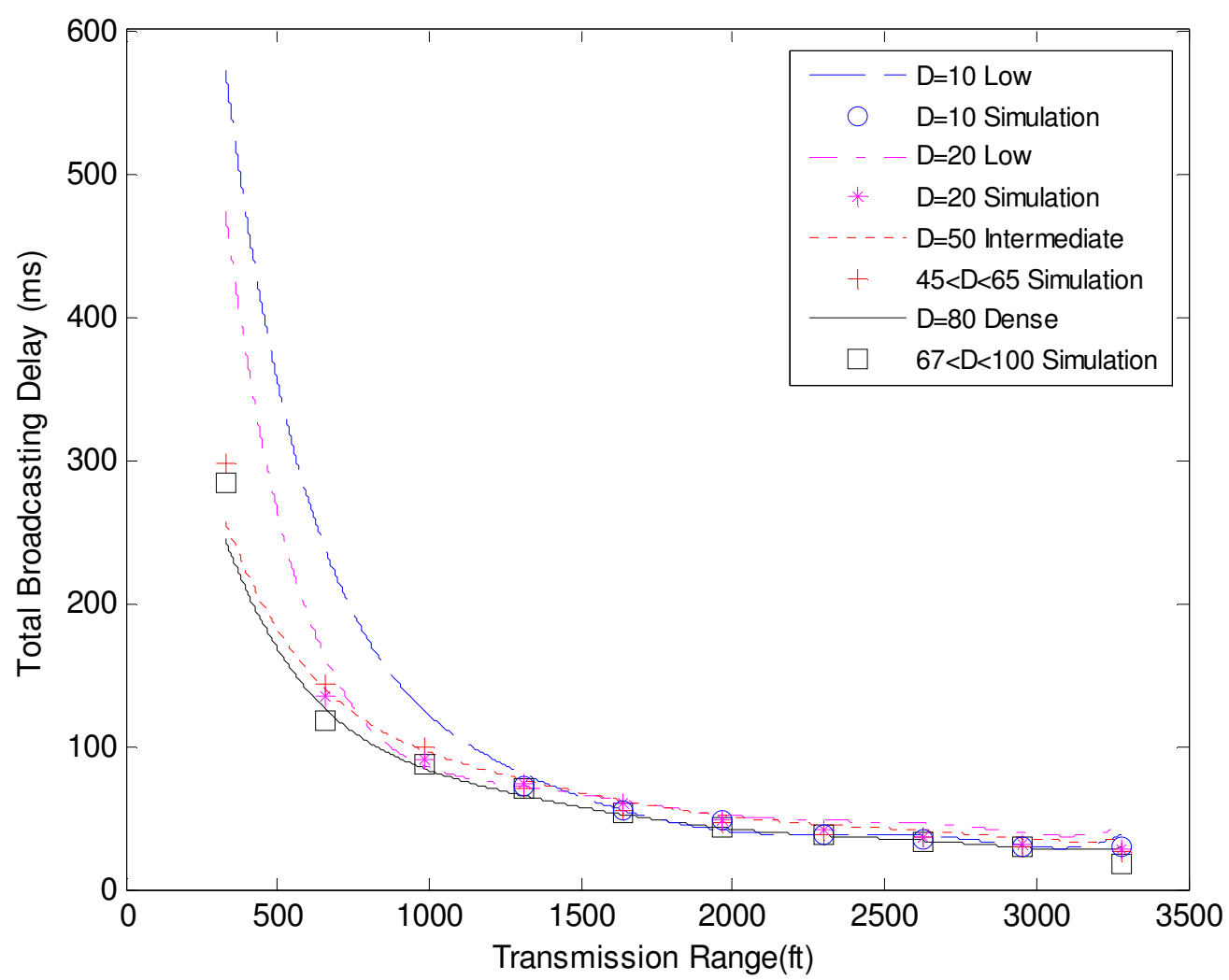

Figure 10. The total broadcasting delay versus cluster size (represented by transmission range) for different traffic flow densities $(10,20,50$, and $80 \mathrm{veh} / \mathrm{ml} / \mathrm{lane})$

gateway and that between neighboring gateways. It is observed that an increase in the cluster size for high and intermediate traffic densities does not change the delay greatly. In fact, the curve for high densities in Figure 10 reach saturation much faster (i.e., at a smaller $R$ ) than that for low densities, due to less likely disconnections for a higher density, and hence a smaller delay. It should be mentioned that the curves for analytical results in Figure 10 are fitted to a 6th order polynomial. Also, in (4.9), as the transmission range changes, the number of nodes between the $\mathrm{CH}$ and its gateway changes, which changes the number of summed exponential terms. As a result, the total delay fluctuates on a small scale with the transmission range. 


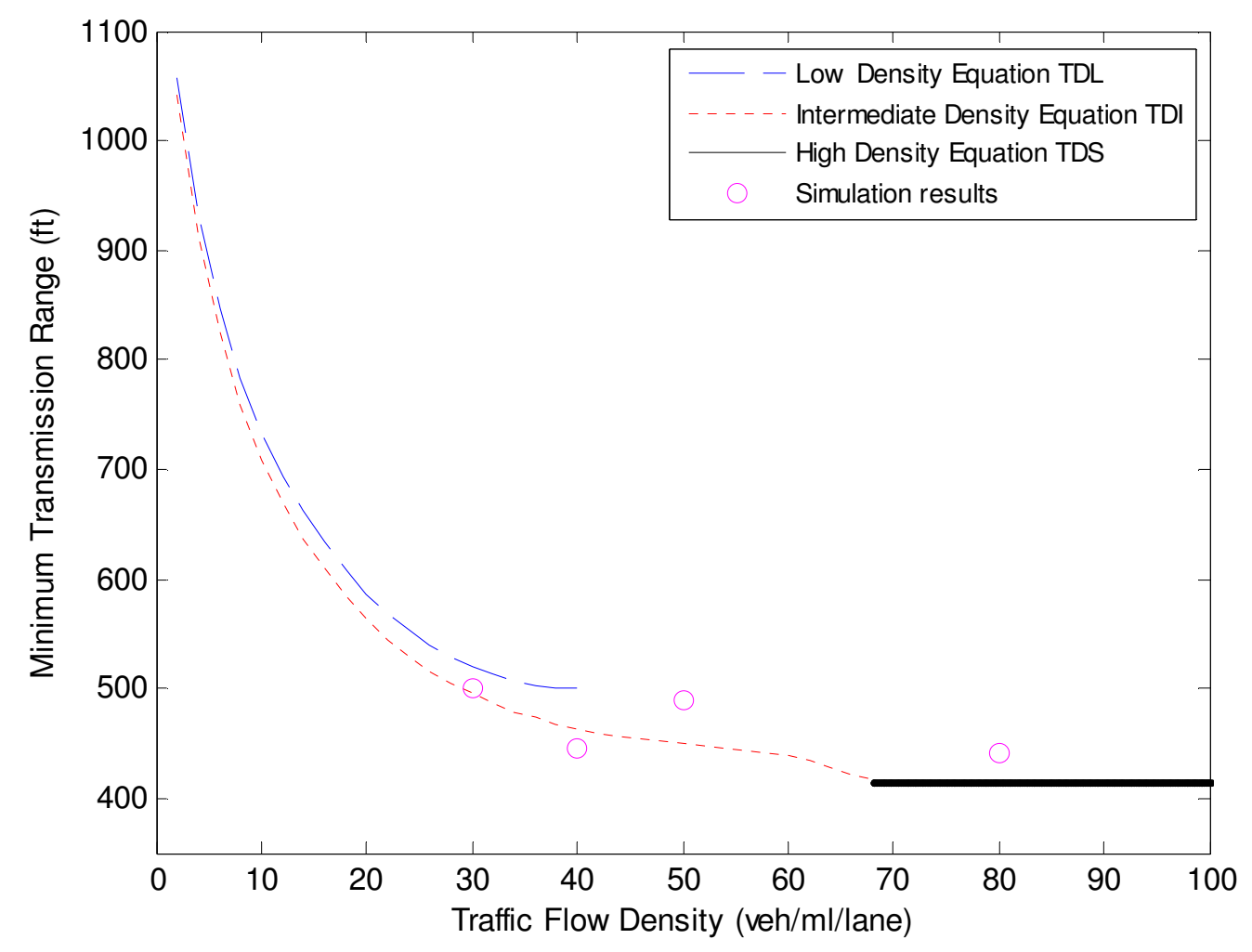

Figure 11. The lower bound cluster size versus traffic flow density for the three traffic flow states

Figure 11 shows the minimum transmission range versus the traffic flow density, in order to satisfy a message broadcasting delay of $200 \mathrm{~ms}$. As the traffic density increases, the transmission range can be reduced while meeting the delay requirement. The gap shown in Figure 11 which results from using different equations is due to the effect of Pp. It is noted that the range changes sharply at a very low density $(<10 \mathrm{veh} / \mathrm{ml} / \mathrm{lane})$. However, such a low density is not the focus of this work, since clustering targets high node density networks, while simple flooding is more suitable for a very low node density situation. Figure 12 plots the total broadcasting delay versus traffic flow density for several transmission ranges. As the density increases, the total delay decreases for the same transmission range. However, as the 


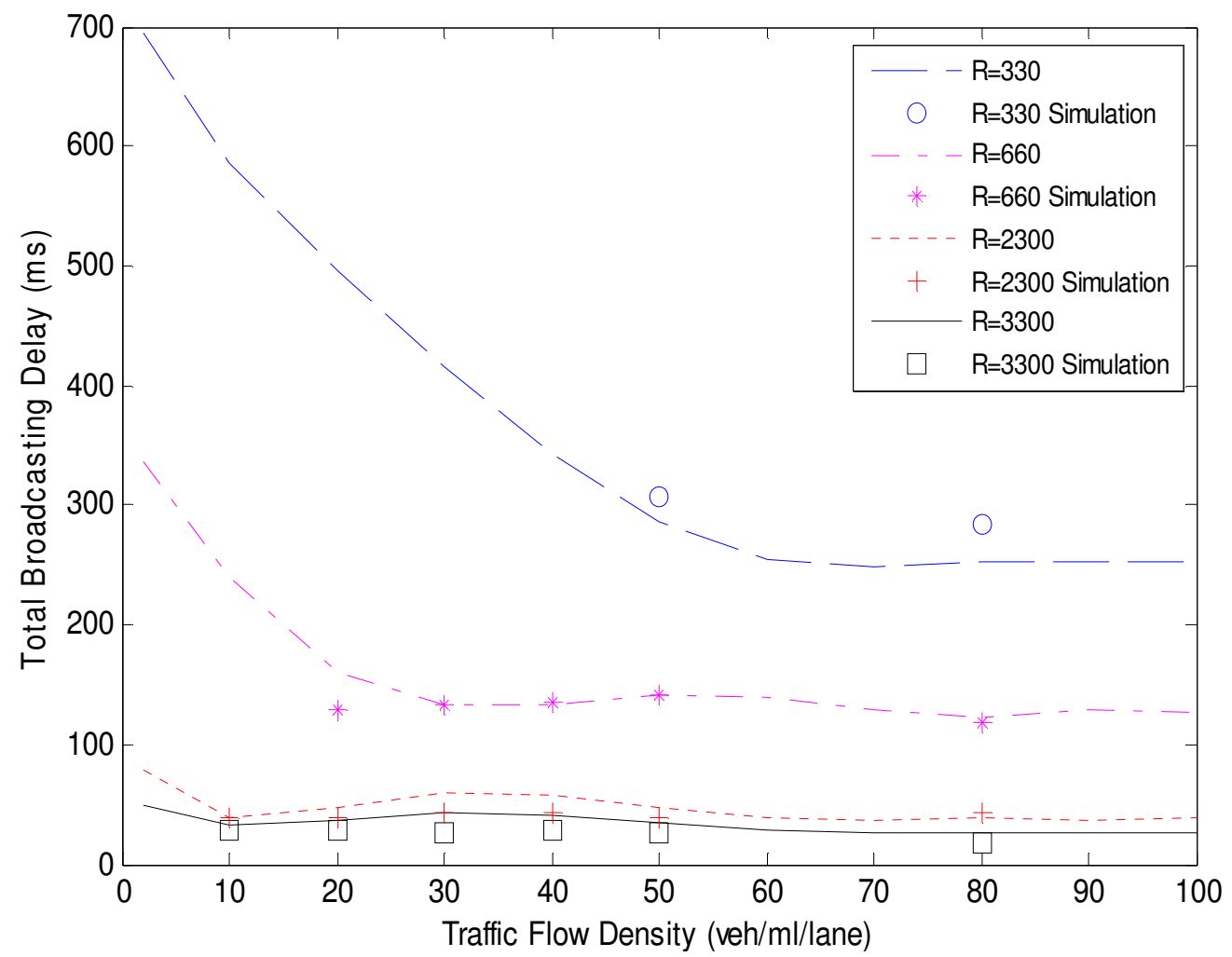

Figure 12. Total broadcasting delay versus traffic flow density for different transmission ranges $(330,660$, 2300 , and $3300 \mathrm{ft}$ )

density increases to a certain threshold, the delay remains almost constant with a further increase in the density. This density threshold decreases as $R$ increases, due to higher chances of node connections. The gaps between the curves in Figure 12 represent the delay reduction gained by increasing $R$. Note that this gain reduces as the transmission range increases. For example, increasing $R$ from $2300 \mathrm{ft}$ to $3300 \mathrm{ft}$ does not give any significant impact on reducing the delay. This shows the importance of determining the design parameter $R$. 


\subsubsection{Simulation Results}

Simulations are carried out for performance evaluations and model verifications. As the nature of VANETs makes it difficult to test the models and analysis in a real networking environment because of several reasons such as the high cost of setting up a test bed of the outdoor environment, difficulty in deploying many vehicles, and the complexity of implementing all possible environmental situations. As a result we resort computer simulation for performance evaluations.

Despite recent research attention on VANETs, few VANET-specific simulators have been proposed in literature $[30,31]$. The reason that not much efforts have been paid to develop a complete VANET-specific simulator is the application specific nature of VANETs. It is difficult to cover all possible application and traffic scenarios. Hence, a possible way is to use already existing network simulators and integrate the node movement scenario and network setup to mimic the behavior of vehicles on the road environment under study. As mentioned earlier, MANET mobility models are not valid for VANETs and hence researchers have put a great effort to create realistic mobility models that can be plugged into MANET simulators.

Many VANET traffic generators have been proposed [34-37], however the mobility scenarios are based on some particular mathematical model, or some pre-chosen speed range and pattern. To ensure the closeness of vehicle movements to real traffic scenarios, we use real highway data sets provided by Next Generation Simulation (NGSIM) online database [36], "U.S Highway 101" and "new I-80", to obtain mobility samples for both intermediate and high density. The data sets are available for relatively short sections ( 2000 ft) but for a 
very long time interval (45 minutes). To cover the broadcasting zone, we build the samples by combining the data from different time intervals to construct scenarios for 15.5 miles. The data sets are collected from seven lane highways, and we choose one of three consecutive lane combinations (lanes 1,2 and 3 or lanes 2, 3 and 4). For consistency, any vehicle switches outside of those three lanes is excluded from our samples. Also, we avoid using lane 5 as it includes vehicles arriving from the on-ramp "lane 6" and the off-ramp "lane 7". For a low traffic density, because of the lack of highway measurement data, we generate random mobility scenarios in simulation. The low density samples have shifted exponential gaps as specified in (3.7) and constant speeds (50,62 and $75 \mathrm{mph}$ ) for the right lane, middle lane, and left lane, respectively. We conduct our simulations using ns-2 [35]. Initially, we modify the distributed clustering algorithm (DCA) [13] to represent the clustered infrastructure assumed in the system model.

The clustering algorithm is used only for constructing the initial clustered infrastructure. The resulting backbone is used as an input to the broadcasting scheme. On the other hand, any cluster maintenance such as re-election of gateways is implemented in the broadcasting scheme itself. Simulations are carried out without any cluster segregation. Since cluster segregations are more likely to occur for a low traffic density and/or for small transmission ranges, no simulation results are obtained for such scenarios. However, to evaluate the proposed model for a low traffic density, Error! Reference source not found. shows clearly how close the simulation results to the analytical results obtained using our model. To avoid cluster segregation, simulations for this traffic flow density could only be performed for a 


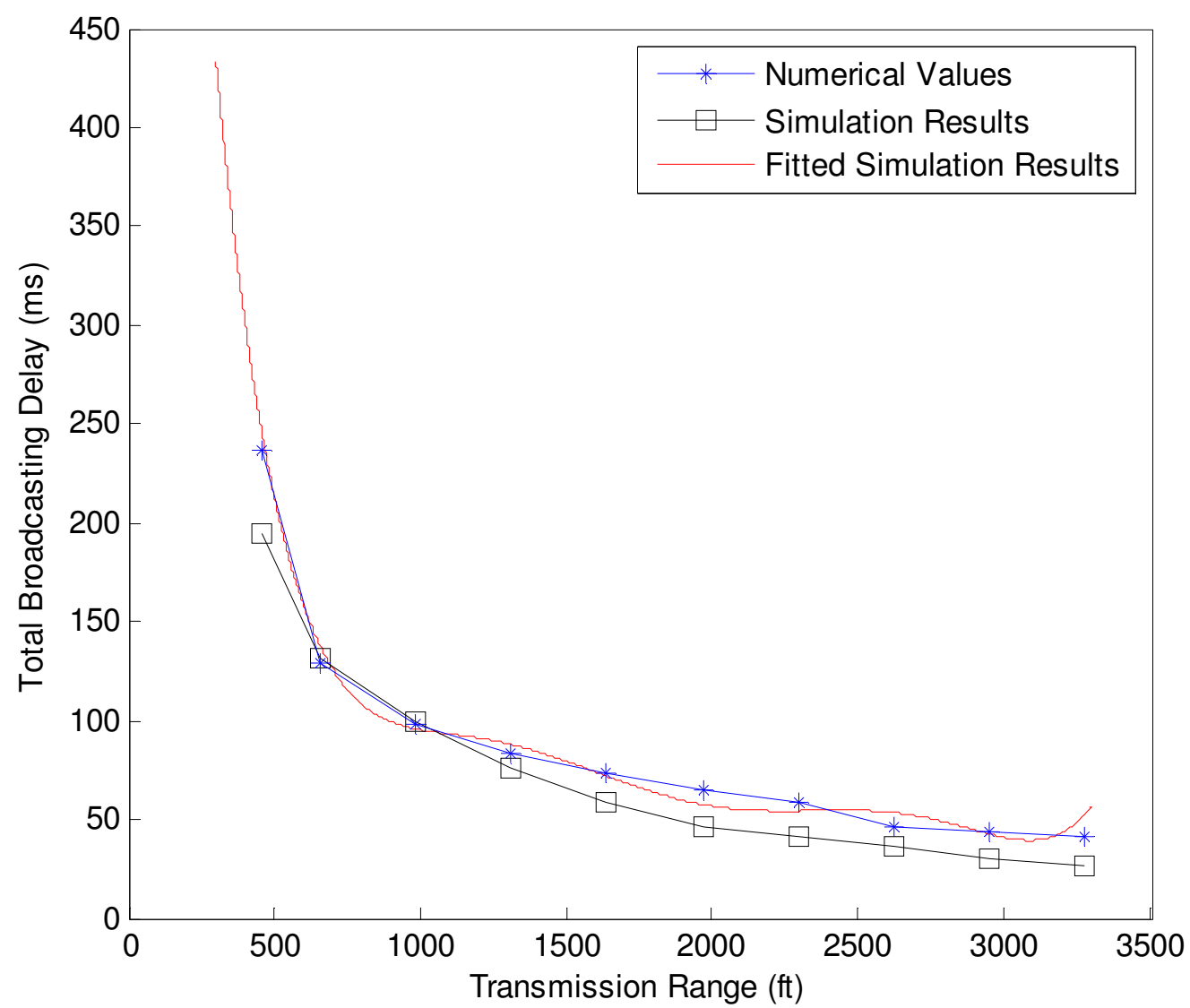

Figure 13 Comparison between simulation results and numerical values for the total broadcasting delay versus cluster size (represented by transmission range) for a density of $30 \mathrm{veh} / \mathrm{ml} / \mathrm{lane}$

transmission range of more than 460 feet. Hence, we plot the fitted values to a 6 th order polynomial and note the closeness to the numerical values.

Simulation results are shown as discrete points in Figures 10, 11 and 12 as well, which verify clearly the accuracy of our proposed model. It is also noticeable that the errors in the results are sometimes random while they follow certain pattern in other parts of the plots. The variance, in the pattern of errors, goes back to the difference in the type of traffic data used to represent the mobility of nodes. Notice the errors are somehow random when 
using real highway data while they tend to follow a certain pattern when using generated data, which is expected.

During simulations, we calculate the number of re-elections that occur because of disconnections in the clustering backbone. It is noted that the number of re-elections increase as the transmission range reduce for the same density. Also the likelihood of re-elections increases for a smaller traffic density. This shows the effect of traffic flow condition on the broadcasting delay. 


\section{Chapter 5}

\section{Conclusions and Future Work}

\subsection{Conclusions}

Reliable and fast transmission of safety messages among vehicles moving in an ad hoc manner has been the focus of ITS for some years now. How fast should a message be delivered to each vehicle in the danger zoon? Are the communication protocol parameters set to adhere to the delay requirements? This research is a step from a certain perspective towards providing answers for those questions.

This research is concerned with two aspects in VANETs. The first is modeling, where we present a mobility-aware mathematical model for broadcasting delay of emergency messages in cluster based VANETs. This is achieved by utilizing highway traffic models developed in civil engineering which provide a basis for analyzing communication delays. Since the traffic flow density plays a key role in the traffic modeling, we derive expressions for the broadcasting delay based on three (low, intermediate, and high) traffic density regions.

The second aspect is the study of delay for emergency messaging in VANETs. We investigate the effect of traffic flow density and node transmission range on the total broadcasting delay. The results of investigation show that the network control and 
performance parameters are dependent on the traffic density. In addition, the minimum cluster size that achieves acceptable message delivery latency has been investigated. It is shown that communication protocol parameters should be a function of traffic density level. NS2 simulations, based on data collected from real highways, are conducted to verify the proposed model.

\subsection{Future Work}

This research provides an elemental framework for emergency messaging delay in VANETs. There are many open areas and issues that should be studied. The following are potential future research directions:

- The main focus of this research is modeling broadcasting delay for any ZOR length and investigating it for a particular ZOR. In reality, knowing the delay requirement for every vehicle depends on two factors, the gap length to the leading vehicle and vehicle location from the incident vehicle. Larger gaps means more time for vehicles to react in order to avoid an incident ahead. The location of the vehicle from the source node, decides the number of hops a safety message propagates to reach the vehicle. As the message propagates, the accumulated reaction time of the drivers would cause that the last vehicle to have less time to react [5]. It would be quite interesting and beneficial to determine the relation between the delay requirement and the distance from the source node in terms of number of hops. 
- It should be useful to study the effect of driver behaviors and lane changes on the message propagation. Some studies have been conducted in civil engineering to model lane changing and driver's aggressiveness [38]. Those valuable studies can be used as a base for this approach.

- In this research, we consider a given constant ZOR in our broadcasting delay investigation. In reality some several kilometer pile-ups have indeed occurred [2]. It would be interesting to determine the size of ZOR for emergency message broadcasting and to investigate its dependency on the traffic flow density. 


\section{References}

[1] National safety council, "Report on injuries in america," Injury facts, 2009 Edition. Available at: http://www.nsc.org/lrs/injuriesinamerica08.aspx.

[2] The local "Dozens injured in 259-car autobahn pileup," July, 2009. Available at: http://www.thelocal.de/national/20090720-20701.html.

[3] Ministry of Transportation "Intelligent transportation systems," 2009. Available at: http://www.mto.gov.on.ca/english/engineering/its/index.shtml.

[4] General Motors, “Automatic crash response,” 2009. Available at: http://www.onstar. com/us_english/jsp/plans/acr.jsp.

[5] C. Young, B. Chang, S. Chen, and L. Wang, "A highway traffic simulator with dedicated short range communications based cooperative collision prediction and warning mechanism,” Proc. IEEE Intelligent Vehicles Symposium. June 2008.

[6] Y. Zang, L.Stibor, H.-J. Reumerman, and H. Chen, "Wireless local danger warning using inter-vehicle communications in highway scenarios," Proc. 14th European Wireless Conf. 2008.

[7] N. Wisitpongphan, P. F. B. Mudalige, V. Sadekar, and O. Tonguz, "Routing in sparse vehicular ad hoc wireless networks," IEEE. Selected Areas Communications, Vol. 25, No. 8 pp.1538-1556, Oct. 2007. 
[8] G.H. Mohimani, F. Ashtiani, A. Javanmard, and M. Hamdi, "Mobility modeling, apatial traffic distribution, and probability of connectivity for sparse and dense vehicular ad hoc networks," IEEE Trans. Vehicular Technology, to appear.

[9] A.D. May, Traffic flow fundamentals. Englewood Cliffs, N.J.: Prentice Hall, 1990.

[10] S. Yousefi, M. S. Mousavi, and M. Fathy, "Vehicular ad hoc networks (VANETs): challenges and perspectives," Proc. 6th Int. Conf. ITS Telecommunications, pp. 761766, June 2006.

[11] M. L. Sichitiu and M. Kihl, "Inter-vehicle communication systems: a survey," IEEE Communication Surveys and Tutorials, Vol. 10, No. 2, pp. 88-105, July 2008.

[12] H. Hartenstein and K. P. Laberteaux, "A tutorial survey on vehicular ad hoc networks," IEEE Communications Magazine. Vol. 46, No. 6, pp. 164-171, June 2008.

[13] S. Basagni, M. Mastrogiovanni, A. Panconesi, and C. Petrioli, "Localized protocols for ad hoc clustering and backbone formation: a performance comparison," IEEE Trans. Parallel and Distributed Systems, Vol. 17, pp.292-306, April 2006.

[14] Y. Wei, J. Heidemann and D. Estrin, "An energy-efficient MAC protocol for wireless sensor networks," Proc. $21^{\text {st }}$ Annual Joint Conf. of the IEEE Computer and Communications Societies. Vol. 3, pp. 1567-1576, 2002.

[15] A. Chungtragarn and P. Keeratiwintakorn, "Finding optimized transmission power for clustering of S-MAC protocol in WSNs," Asia-Pacific Conf. on Communications, pp. $163-166,2007$. 
[16] J.Y. Yu and P.H.J. Chong, "A survey of clustering schemes for mobile ad hoc networks," IEEE Communications Surveys and Tutorials, Vol. 7, No. 1, pp. 32-48, 2005.

[17] P. Basu, N. Khan, and T. D. C. Little, "A mobility based metric for clustering in mobile ad hoc networks," Proc. IEEE Int. Conf. Distributed Computing Systems Workshops, pp. 413-418, Apr. 2001.

[18] R. A. Santos, R. M. Edwards, and A. Edwards, "Cluster-based location routing algorithm for vehicle to vehicle communication," Proc. IEEE Int. Conf. Computer Communications and Networks, pp. 221-226, Oct 2004.

[19] O. Kayis and T. Acarman, "Clustering formation for inter-vehicle communication," Proc. IEEE Conf. Intelligent Transportation Systems, pp.636-641, 2007.

[20] Z. Wang, L. Liu, M. Zhou, and N.Ansari, “A position-based clustering technique for ad hoc intervehicle communication," IEEE Trans. systems, Vol. 38, No. 2, March, 2008.

[21] H. Su and X. Zhang, "Clustering-based multichannel MAC protocols for QoS provisioning over vehicular ad hoc networks," IEEE Trans.Vehicular Technology, Vol. 56, No. 6, Nov. 2007.

[22] K. Ibrahim and M. C. Weigle, "Towards an optimized and secure cascade for data aggregation in VANETs," Proc. $5^{\text {th }}$ ACM Int. Conf. Mobile Computing and Networking, Sept.2008. 
[23] F. Li and Y. Wang, "Routing in vehicular ad hoc networks: A survey," IEEE Vehicular Technology Magazine, Vol. 2, No. 2, pp. 12-22, June 2007.

[24] B. Williams, T. Camp. "Comparison of broadcasting techniques for mobile ad hoc networks," Proc. $3^{\text {rd }}$ ACM Int. symposium Conf. Mobile ad hoc networking and computing, 2002.

[25] Bachir and A. Benslimane, "A multicast protocol in ad hoc networks inter-vehicle geocast," Proc. 57th IEEE Semiannual Conf. Vehicular Technology, vol. 4, pp. 24562460,2003

[26] R.A. Santos, R.M Edwards, L.N. Seed, and A. Edwards, “A location-based routing algorithm for vehicle to vehicle communication," Proc.13th Int. Conf. Computer Communications and Networks, 2004.

[27] M. Durresi, A. Durresi, and L. Barolli, "Emergency broadcast protocol for intervehicle communications," Proc. IEEE 11th Int. Conf. Parallel and Distributed Systems,Vol. 2, pp.402 - 406, July 2005.

[28] J. T. Yu, "Requirements analysis of IP and MAC protocols for dedicated short range communications (DSRC)," Proc. 16th Int.Conf. Wireless Communications, Calgary, Canada, July 2004.

[29] W. R. Chang, H.T. Lin, and B. X. Chen, "TrafficGather: An efficient and scalable data collection protocol for vehicular ad hoc networks" Proc. 5th IEEE Consumer Communications and Networking, pp. 365-369, Jan. 2008. 
[30] M. Piorkowski , M. Raya, A. Lugo, P. Papadimitratos, M. Grossglauser, and J. P. Hubaux, "TraNS: Realistic joint traffic and network simulator for VANETs" ACM SIGMOBILE Mobile Computing and Communications Review, Vol. 12, No. 1, pp. 31-33, 2008.

[31] R. Mangharam, D. S. Weller, D. D. Stancil, R. Rajkumar, and J. S. Parikh, “GrooveSim: A topography-accurate simulator for geographic routing in vehicular networks" Proc. $2^{\text {nd }}$ ACM Int. Workshop Vehicular Ad hoc Networks, Sept. 2005.

[32] F.K. Karnadi, Z. H. Mo, and K-C Lan, "Rapid generation of realistic mobility models for VANET," Proc. IEEE Conf. Wireless Communications and Networking, pp. 2506-2511, 2007.

[33] G. De Marco, M. Tadauchi, and L. Barolli, “CAVENET: Description and analysis of a toolbox for vehicular networks simulation," Proc. IEEE Int. Conf. Parallel and Distributed Systems, Vol. 2, pp. 1-6, Dec. 2007.

[34] David Choffnes and Fabián E. Bustamante, "STRAW - An integrated mobility and traffic model for VANETs," Proc. 10th Int. Command and Control Research and Technology Symposium, June 2005.

[35] The Network Simulator ns-2, http://www.isi.edu/nsnam/ns/

[36] Next Generation Simulation Community Data Sets, U.S department of Transportation, Federal Highway Administration, http://www.ngsim.fhwa.dot.gov/.

[37] K-C. Lan and C-M. Chou, "Realistic mobility models for vehicular ad hoc network simulations," Proc. 8th Int. Conf. ITS Telecommunication, pp. 362-366, 2008. 
[38] A. Kesting, M. Treiber, and D. Helbing, "General lane-changing model MOBIL for car-following models," Transportation Research Record, Vol. 1999, pp. 86-94, 2007. 